Study of the photoelectron and electron momentum spectra of cyclopentene using benchmark Dyson orbital theories

Non Peer-reviewed author version

HUANG, Yanru; Ning, Chuan G.; Deng, Jing K. \& DELEUZE, Michael (2008) Study of the photoelectron and electron momentum spectra of cyclopentene using benchmark Dyson orbital theories. In: PHYSICAL CHEMISTRY CHEMICAL PHYSICS, 10(17). p. 2374-2389.

DOI: $10.1039 / \mathrm{b} 718588 \mathrm{j}$

Handle: http://hdl.handle.net/1942/8259 


\title{
Study of the Photoelectron and Electron Momentum Spectra of Cyclopentene using Benchmark Dyson Orbital Theories
}

\author{
Yan R. Huang ${ }^{1,2}$, Chuan G. Ning ${ }^{1}$, Jing K. Deng* ${ }^{1}$, Michael S. Deleuze*²
}

1. Department of Physics and Key Laboratory of Atomic and Molecular NanoSciences of MOE, Tsinghua University, Beijing 100084, P.R. China

2. Research Group of Theoretical Chemistry, Department SBG, Hasselt University, Agoralaan Gebouw D, B-3590 Diepenbeek, Belgium

*Corresponding authors:

J.K. Deng email: djk-dmp@tsinghua.edu.cn

M.S. Deleuze e-mail: michael.deleuze@uhasselt.be

Abstract: A complete study of the valence electronic structure and related electronic excitation properties of cyclopentene in its $\mathrm{C}_{\mathrm{s}}$ ground state geometry is presented. Ionization spectra obtained from this compound by means of photoelectron spectroscopy (He I and He II) and Electron Momentum Spectroscopy have been analyzed in details up to electron binding energies of $30 \mathrm{eV}$ using one-particle Green's function (1p-GF) theory along with the outer-valence (OVGF) and the third-order algebraic diagrammatic construction [ADC(3)] schemes. The employed geometries derive from DFT/B3LYP calculations in conjunction with the aug-cc-pVTZ basis set, and closely approach the structures inferred from experiments employing microwave spectroscopy or electron diffraction in the gas phase. The 1p-GF/ADC(3) calculations indicate that the orbital picture of ionization breaks down at binding energies larger 
than $\sim 17 \mathrm{eV}$ in the inner-valence region, and that the outer-valence $7 \mathrm{a}^{\prime}$ orbital is also subject to a significant dispersion of the ionization intensity over shake-up states. This study confirms further the rule that OVGF pole strengths smaller than 0.85 foretell a breakdown of the orbital picture of ionization at the $\operatorname{ADC}(3)$ level. Spherically averaged (e, 2e) electron momentum distributions at an electron impact energy of $1200 \mathrm{eV}$ that were experimentally inferred from an angular analysis of EMS intensities have been interpreted by comparison with accurate simulations employing ADC(3) Dyson orbitals. Very significant discrepancies were observed with momentum distributions obtained from several outer-valence ionization bands using standard Kohn-Sham orbitals.

Key words: Orbital imaging, ionization, electronic relaxation, electronic correlation, shake-up, Green's Function Theory, Density Functional Theory

\section{Introduction}

Five-membered unsaturated cyclic molecules such as cyclopentene behave like four-membereds ring for the ring-puckering vibration since the two atoms that are linked by by the more rigid double bond merely move together as one single atom [1]. The cyclopentene molecule represents therefore a very ideal prototype for studying large-amplitude ring-puckering motions in the gas phase [2]. Cyclopentene is thus typically the most thoroughly studied example of a pseudo-four-membered-ring molecule characterized by a double-minimum potential function describing low-frequency puckering vibrations. A number of experiments employing far-infrared [1, 3-5], mid-infrared, [6-17], Raman [14-24], and microwave [25-27] spectroscopy, electron diffraction [28], and various theoretical analyses [29-37] indicate that 
cyclopentene has a non-planar equilibrium structure of $\mathrm{C}_{\mathrm{s}}$ symmetry, with the carbon atom opposite to the double bond being tilted out of the plane of the four other carbon atoms. Cyclic unsaturated hydrocarbons such as cyclopentene $\left(\mathrm{C}_{5} \mathrm{H}_{8}\right)$ have been widely used for the making of ordered organic monolayer films on semiconductor surfaces of crystalline silicon ( $\mathrm{Si}$ ) [38-44], germanium (Ge) [45], diamond (C) [46]. In view of the practical importance of cyclo-addition reactions between unsaturated compounds and semi-conductor surfaces, the main goal of the present work is therefore to expand our understanding of the electronic structure (and wavefunction) of cyclopentene and its relationships with the molecular structure, as well as with its interplay with related electronic excitation properties.

In a first study at Tsinghua University [47] employing Electron Momentum Spectroscopy (EMS) [48-50], the experimentally inferred electronic structure of cyclopentane was assigned from an analysis of the angular dependence of electron-impact (e, 2e) ionization intensities by comparison with theoretical spherically averaged electron momentum distributions that were empirically derived from standard (B3LYP) Kohn-Sham orbitals. It is here worth reminding again that EMS is particularly sensitive to the low momentum (large $r$ ) part of the outermost orbital densities that are important in molecular recognition processes and at the early stages of a cyclo-addition reaction. Attempts were made in this first study for extending the band assignment up to the bottom of the inner-valence region, despite major drawbacks in the modeling such as the restriction of the analysis to one-electron ionization lines, and the uncertainties inherent to using Kohn-Sham orbitals derived from DFT calculations on neutrals molecules for computing electron momentum distributions that formally relate to transition amplitudes between neutral and cationic wave functions. 
Sophisticated quantum mechanical treatments that cope, at least, with electron correlation and relaxation effects, as well as with the dispersion of the ionization intensity over shake-up states arising from configuration interaction effects in the cation [51] are the most basic requirements for reliably assigning (e, 2e) ionization spectra and conduct from these a safe analysis of experimental electron momentum distributions. A number of theoretical studies employing one-particle Green's Function theory [52] along with the third-order algebraic diagrammatic scheme [ADC(3)] scheme [51f, 53] have indeed enlightened the importance of shake-up bands in the outer-valence ionization spectra of unsaturated compounds, such as polyenes [51e, 54], carbon clusters [51f-i], polycyclic aromatic hydrocarbons [51j-o], furane, trans-stilbene [51n], purine and pyrimidine $[51 \mathrm{~m}]$, pyrrole and thiophene [55], whereas further ADC(3) calculations have demonstrated that even saturated hydrocarbons are subject to a strong breakdown of the orbital picture of ionization in the inner-valence region [56]. Most EMS experiments so far have nonetheless been interpreted on the assumption of a one-to-one correspondence between the observed ionization bands and one-electron ionization processes, and are therefore likely to undergo substantial revisions in the future. The interested reader is referred in particular to strong refutations of band assignments drawn for the ionization spectra of stella-2,6-dione [57] or 1,3-butadiene [58] from EMS experiments, which indicate that it is indeed impossible to reliably assign highly congested (e, 2e) ionization spectra by resorting only to standard Kohn-Sham (or Hartree-Fock) orbital energies and to the related electron densities. In contrast with DFT, one-particle Green's Function theory also provides a formally exact theoretical framework for interpreting electron momentum distributions inferred from EMS experiments at high electron impact energies, through the interplay of Dyson orbitals. Recent applications of 
the 1p-GF/ADC(3) approach on difluoromethane [59], dimethoxymethane [60] and water [61] and comparison with DFT results demonstrate indeed that this Many Body Green's Function approach provides overall superior insights into the experimental momentum distributions.

Experimental studies of the valence electronic structure of cyclopentene are rather scarce. Besides the (e, 2e) ionization spectrum recorded by Ren et al [47] at an electron impact of 1200 $\mathrm{eV}$, these comprise only a few photoelectron studies [62] employing $\mathrm{He}$ I or He II radiation beams. The available theoretical data available for this spectra are very limited too [47], and do not go beyond one-electron ionization energies obtained from HF or KS orbital energies, under the assumption of Koopmans's theorem and a DFT extension of it, and using the Outer Valence Green's Function (OVGF) approach [63]. The present 1p-GF/ADC(3) study of the valence ionization spectrum of cyclopentene along with an analysis of the previously inferred (e, 2e) electron momentum distributions on the ground of 1p-GF/ADC(3) Dyson orbitals enables us to considerably improve, both from a qualitative and quantitative viewpoint, our understanding of the electronic structure of cyclopentene and its interplays with electronic correlation and relaxation effects in ionization experiments.

\section{Theoretical background for ionization phenomena and electron momentum spectroscopy}

The basic principle of EMS [48-50, 64] is an ionization plus electron scattering process initiated by electron collision, i.e. a so-called $(\mathrm{e}, 2 \mathrm{e})$ reaction. The gas phase target molecules are ionized by impact with a high energy electron beam, whereas the two outgoing electrons are selected and then detected in coincidence. The experimental set-up is based on a symmetric non-coplanar geometry, i.e. the two outgoing electrons are selected at equal energies and polar 
angles $\left(\theta_{1}=\theta_{2}=45^{\circ}\right)$ relative to the incoming electron beam direction. Plots of differential (e, 2e) ionization cross section versus the molecular recoil momentum $\vec{q}$ are obtained for each resolved ionized state of the target molecule by monitoring in coincidence the dependence of the electron impact ionization cross sections upon the azimuthal angle $(\phi)$ between the two outgoing electrons, which in our experimental set-up varies from $-30^{\circ}$ to $30^{\circ}$ around the incident beam axis. For a clear knock-down collision process, the molecular recoil momentum $\vec{q}$ can be in turn directly related to the momentum of the electron $(\vec{p})$ prior to the ionization process, via $\vec{q}=-\vec{p}$. Assuming a binary (e, 2e) encounter, and thus, a high electron impact energy, a high momentum transfer and a negligible kinetic energy transfer to the residual ion, the initial momentum of the knocked-out electron is indeed related to the azimuthal angle $\phi$ by [50]

$p=\left[\left(2 p_{1} \cos \theta-p_{0}\right)^{2}+4 p_{1}^{2} \sin ^{2} \theta \sin ^{2}\left(\frac{\phi}{2}\right)\right]^{\frac{1}{2}}$

where $p_{1}$ and $p_{0}$ are the momenta of the outgoing and incident electrons, respectively. Under the assumptions of the Born, binary encounter, and plane wave impulse approximations $[48,49$, $64 \mathrm{~b}, 50]$, the $(\mathrm{e}, 2 \mathrm{e})$ ionization cross sections are in turn directly proportional to structure factors derived as the Fourier transforms of Dyson orbitals for the ionization channels under consideration:

$$
\sigma_{n}=K \int\left|g_{n}(\omega, p)\right|^{2} d \Omega
$$

where $\omega$ and $p$ represent the spin and momentum of the electron prior to ionization. In the above equation, $\int d \Omega$ denotes the spherical average over all orientations of the target molecule. Using spin-space coordinates $\mathbf{x}=(\omega, \vec{r})$, Dyson spin-orbitals orbitals are defined [59, 65] as partial overlaps between the initial neutral ground state and final ionized states:

$$
g_{n}(\mathbf{x})=\sqrt{N} \int \Psi_{n}^{N-1}\left(\mathbf{x}_{1}, \mathbf{x}_{2}, \cdots, \mathbf{x}_{N-1}\right) \Psi_{0}^{N}\left(\mathbf{x}_{1}, \mathbf{x}_{2}, \cdots, \mathbf{x}_{N-1}, \mathbf{x}\right) d \mathbf{x}_{1} d \mathbf{x}_{2} \cdots d \mathbf{x}_{N-1}
$$


with $N$ the number of electrons. These spin-orbitals $g_{n}(\mathbf{x})$ can therefore be regarded as effective orbitals for the holes created in the cationic states $\left|\Psi_{n}^{N-1}\right\rangle$, which account both for ground state correlation and dynamical relaxation effects, as well as for the dispersion of the ionization intensity over states relating to excited (shake-up) electronic configurations of the cation: by definition, the norm of Dyson orbitals is smaller than one. Note that the above equations (2) and (3) provide a formally exact depiction of $(\mathrm{e}, 2 \mathrm{e})$ cross sections in the high-energy limit $\left(E_{0}>>1\right.$ $\mathrm{keV}$ ) ensuring the so-called EMS conditions [48, 49, 64, 50]

As EMS, one-particle Green's Function (1p-GF) theory [52] enables a direct mapping, and this within an exact many-body framework, of vertical ionization energies and Dyson orbitals. For an $N$-particle system with a non-degenerate ground state $\left|\Psi_{0}^{N}\right\rangle$, the spectral representation of the advanced component of the one-particle Green's function has the following definition $[52 \mathrm{a}, 52 \mathrm{~b}, 71 \mathrm{~b}$,

$$
G_{i j}(\omega)=\sum_{n \in(N-1)} \frac{x_{i}^{(n)} x_{i}^{(n)^{*}}}{\omega+I P_{n}-i O^{+}}
$$

where $I P_{n}$ represents the ionization potential corresponding to the $N$-particle system. It is immediately apparent that the positions of lines in the ionization spectrum are given from the location of the poles of the Green's function in the complex energy plane [65a]. The corresponding residues relate to products of Feynman-Dyson transition amplitudes:

$x_{i}^{(n)}=\left\langle\Psi_{n}^{N-1}\left|a_{i}\right| \Psi_{0}^{N}\right\rangle, \quad \forall n \in\{N-1\}$

The probability for a given ionic state $\left|\Psi_{n}^{N-1}\right\rangle$ is given by

$$
\Gamma_{n}=\left\|\left\langle\Psi_{n}^{N-1} \mid \Psi_{0}^{N}\right\rangle_{N-1}\right\|^{2}=\sum_{i}\left\|x_{i}^{(n)}\right\|^{2}
$$

which corresponds to the pole strength $\left(\Gamma_{n}\right)$, alternatively defined as $\operatorname{tr}[G(\omega)]$ at $\omega=-I_{n}$. 
$\Gamma_{n}$ provides also the factor required to normalize the Dyson orbital related to state $\left|\Psi_{n}^{N-1}\right\rangle$, which is defined as a linear combination of the HF orbital $\left|\varphi_{i}\right\rangle$, with the Feynman-Dyson transition amplitudes as weight coefficients [52a, 52b, 71b]

$$
g_{n}(x)=\frac{1}{\sqrt{\Gamma_{n}}}\left\langle\Psi_{n}^{N-1} \mid \Psi_{0}^{N}\right\rangle_{N-1}=\frac{1}{\sqrt{\Gamma_{n}}} \sum_{i} x_{i}^{(n)} \varphi_{i}(x)
$$

\section{Computations}

The geometry of cyclopentene has been optimized using the nonlocal hybrid and gradient corrected Becke-three-parameter Lee-Yang-Parr functional (B3LYP) [66, 67] in conjunction with Dunning's correlation consistent polarized valence basis set of triple- $\zeta$ quality basis augmented by a set of diffuse $\{\mathrm{s}, \mathrm{p}\}$ functions on the hydrogen atoms together with a set of diffuse $\{\mathrm{s}, \mathrm{p}, \mathrm{d}\}$ functions on the carbon atoms (aug-cc-pVTZ) [68]. This approach is known to provide equilibrium geometries and related vibrational properties of quality comparable to that achieved at the benchmark $\operatorname{CCSD}(\mathrm{T})$ theoretical level [69]. All HF, DFT, OVGF, CCSD(T) calculations have been performed using GAUSSIAN 98 [70]. The ADC(3) calculations have been carried out using the original 1p-GF/ADC(3) package of programs, interfaced to GAMESS [71]. This package incorporates a band-Lanczos [72] "pre"- diagonalisation of the block matrices pertaining to the $2 \mathrm{p}-1 \mathrm{~h}$ shake-on states into a pseudo-electron attachment spectrum, prior to a complete block-Davidson diagonalisation [73] of the so-reduced ADC(3) secular matrix. With this diagonalization procedure, all eigen-values of the ADC(3) secular matrix with pole strengths equal to (or larger than) 0.005 could be recovered up to electron binding energies of $\sim 32 \mathrm{eV}$. The assumption of frozen core electrons has been used throughout and the full molecular symmetry point group has been exploited. At the self-consistent field level, the 
requested convergence on each of the elements of the density matrix was fixed to $10^{-10}$. The 1p-GF/ADC(3) calculations described in the present work have been carried out using Dunning's correlation-consistent polarized valence basis set of double- $\zeta$ quality (cc-pVDZ) [74] and Dunning's DZP+ basis sets (namely, a double- $\zeta$ basis set with polarization and diffuse functions) [75].

The ionization spectra presented in the sequel have been simulated using as convolution function a combination of a Gaussian and a Lorentzian with equal weight (Voigt profile) and a constant full width at half of maximum parameter (FWHM) of $0.6 \mathrm{eV}$. The parameter has been selected in order to enable comparisons with available experimental data obtained by means of He I and He II radiation photoelectron spectroscopy [62]. Spherically averaged orbital momentum distributions have been generated from the output of $1 \mathrm{p}-\mathrm{GF} / \mathrm{ADC}(3)$ or DFT calculations using the MOMAP program by Brion and co-workers [76], a homemade interfaces and G03-NEMS [61, 77]. In order to enable physically meaningful comparisons with the experimental momentum profiles, the consequences of the finite acceptance angles $\left(\Delta \theta= \pm 0.6^{\circ}\right.$ and $\left.\Delta \phi= \pm 1.2^{\circ}\right)$ in our experimental set up on the electron momentum resolution $(\Delta p \sim 0.1$ a.u. $)$ were accounted for by resorting to Monte Carlo methods [61,78].

The measured ionization intensities are not absolute values, and the related electron momentum distributions need therefore to be renormalized onto an absolute scale. In the present work, all experimental electron momentum distributions have been rescaled using a constant renormalization factor obtained by comparing experimental results with $\mathrm{ADC}(3) / \mathrm{DZP}+$ electron momentum distributions for the Highest Occupied Molecular Orbital (HOMO, 12a'(1)). The rationale here is that this orbital is very well separated from other valence ionization bands in 
PES [62] and EMS [47] electron binding energy spectra and provides therefore the best reference for a global intensity rescaling.

\section{Results and discussion}

\section{Molecular geometry}

The optimized B3LYP/aug-cc-pVTZ molecular geometry of cyclopentene in its puckered form $\left(\mathrm{C}_{\mathrm{s}}\right.$, Fig. 1) is compared in Table 1 with the experimentally available parameters and results obtained from other molecular orbital calculations $[79,80]$. In general, our results for the bond lengths and bond angles are found to be in good agreement with the experimental data derived from microwave and electron diffraction, in spite of some experimental uncertainties. Table 1 indicates nonetheless that the puckering angle $\varphi$ is rather strongly dependent upon the employed theoretical model and basis set. The value of $13.6^{\circ}$ computed [81] at the HF level and without polarization functions in the basis set, thus without inclusion of electron correlation and a sufficiently flexible basis set, is more than doubtful. Molecular Mechanics (MM) calculations employing the so-called orthodontic force model [82], the consistent force field [83], the $2^{\text {nd }}$ generation force field by Allinger (MM2) [84], the scaled quantum mechanical force field [85], and the $4^{\text {th }}$ generation force field by Allinger (MM4) [79] lead to puckering angle of $19.0^{\circ}$ [86], $21.4^{\circ}[87], 23.0^{\circ}[88], 23.4^{\circ}[85]$, and $24.6^{\circ}[79]$, respectively. Depending on the employed basis set, second-order Møller/Plesset perturbation theory (MP2) yields a slightly larger puckering angle $26.3^{\circ}$ [80]. On the experimental side, a puckering angle of $23.3 \pm 1,22.3 \pm 2$ and $29.0 \pm 2.5^{\circ}$ is inferred from infrared [1], microwave [26,27] and electron diffraction experiments [28], 
respectively, and a value of $26^{\circ}$ has been reported from far-infrared and Raman data [31]. This comparison corroborates the view that, due to shortcomings of the employed functional in the asymptotic region, and limitations of the description of through-space interactions and steric repulsions, B3LYP calculations may somehow drive the molecular structure a little too strongly towards planarity. A further example of the implications of the shortcomings of DFT in describing non-bonded interactions is discussed in a comparative study of the molecular structure and rotational barriers of trans-stilbene using Molecular Mechanics, Density Functional Theory and various many-body approaches up to the confines of non-relativistic quantum mechanics [89]. The obtained puckering angle $\left(19.4^{\circ}\right)$ presented in this work compares nonetheless more than favorably ( $3^{\circ}$ error only) with the results of most experimental structural determinations. For the sake of consistency with many other studies employing Electron Momenta Spectroscopy, we have been willing therefore to retain the B3LYP/aug-cc-pVTZ geometry for performing our analysis on cyclopentene.

At the HF/aug-cc-pVTZ level, cyclopentene, in its most stable puckered form, exhibits a $\mathrm{C}_{\mathrm{s}}$ symmetry point goup and has the following inner and outer valence shell electronic configurations:

inner valence shell : $\left\{\left(4 \mathrm{a}^{\prime}\right)^{2}\left(5 \mathrm{a}^{\prime}\right)^{2}\left(3 \mathrm{a}^{\prime \prime}\right)^{2}\left(6 \mathrm{a}^{\prime}\right)^{2}\left(4 \mathrm{a}^{\prime \prime}\right)^{2}\right\}$, outer valence shell : $\left\{\left(7 a^{\prime}\right)^{2}\left(8 a^{\prime}\right)^{2}\left(5 a^{\prime \prime}\right)^{2}\left(9 a^{\prime}\right)^{2}\left(6 a^{\prime \prime}\right)^{2}\left(10 a^{\prime}\right)^{2}\left(11 a^{\prime}\right)^{2}\left(7 a^{\prime \prime}\right)^{2}\left(12 a^{\prime}\right)^{2}\right\}$. The corresponding molecular orbitals are displayed in Fig. 2

\section{Valence ionization spectra}

The results of our ADC(3) calculations are displayed in Figures $3 \mathrm{c}$ and $\mathrm{d}$ in the form of spike spectra and convoluted densities of states, and compared with the $\mathrm{He}$ I and $\mathrm{He}$ II 
photoelectron spectra (PES) by Bieri et al [62a] and Wiberg et al [62b] in Figures 3a and 3b, respectively. The convolution has been performed using as spread function a combination of a Gaussian and Lorentzian with equal weight, a FWHM parameter of $0.6 \mathrm{eV}$, and by simply scaling the line intensities according to the computed ADC(3) pole strengths. In spite of the neglect of the varying influence of molecular orbital cross sections, the theoretical simulations based on $\mathrm{ADC}(3) / \mathrm{cc}-\mathrm{pVDZ}$ and DZP+ calculations nicely reproduce the positions of the one-electron ionization and shake-up bands in the He I and He II spectra. Both simulations exhibit peaks located at 9.13, 12.24, 13.27, 13.86, 16.02, $16.5617 .64,19.18$, and $22.74 \mathrm{eV}$ (see Fig.3 c and d). The spike and convoluted ADC(3) spectra displayed in Figure 3 reflect the partition of the valence electronic structure of cyclopentene into 5 inner-valence levels $\left(\mathrm{C}_{2 \mathrm{~s}}\right)$ and 9 outer-valence levels $\left(\mathrm{C}_{2 \mathrm{p}}, \mathrm{H}_{1 \mathrm{~s}}\right)$, at electron binding energies above and below $\sim 17 \mathrm{eV}$, respectively. A summary of the available orbital binding energies from the PES and EMS data, as well as our orbital assignment, are given in Table 2.

The first ionization band located at $9.2 \mathrm{eV}$ in the photoelectron spectra corresponds to the removal of one electron from the HOMO (highest occupied molecular orbitals) level 12a' (1). This band lies at considerably lower electron binding energies than the other valence shell orbitals, and is thus very well isolated and resolved. This was not entirely unexpected since the $12 \mathrm{a}^{\prime}$ orbital derives essentially from the localized $\pi(\mathrm{C}=\mathrm{C})$ double bond contribution (Fig.2). In addition, it is immediately apparent from the PES measurements that this band has a sharp vibrational onset followed by a pronounced vibrational structure. For most cycloalkenes molecules, the associated frequency is on the order of $1300-1500 \mathrm{~cm}^{-1}[62 \mathrm{~b}]$ and can only be attributed to a localized stretching mode associated to the $\mathrm{C}=\mathrm{C}$ bond. 
Figure 2 indicates the development of rather strong $\sigma\left(\mathrm{C}_{2 \mathrm{p}}-\mathrm{C}_{2 \mathrm{p}}\right)$ bonding interactions between the out-of-plane carbon atom $\mathrm{C}_{5}$ and its neighbours, $\mathrm{C}_{3}$ and $\mathrm{C}_{4}$. The correponding ionization line therefore appears at a much higher ionization energy (Figures $3 \mathrm{c}, \mathrm{d}$ ), and is subject to a stonger vibrational broadening (Figure 3a), than the $\pi$-level associated to orbital 12a' (1) . Running further towards higher binding energies, the next 7 orbitals that we encounter $\left(11 a^{\prime}, 10 a^{\prime}, 6 a^{\prime \prime}, 9 a^{\prime}, 5 a^{\prime \prime}, 8 a^{\prime}, 7 a^{\prime}\right)$ merely relate to combinations of $\mathrm{C}_{2 p}$ and $\mathrm{H}_{1 \mathrm{~s}}$ orbitals. The theoretical vertical ionization energies presented in Table 2 show that although the order of ionization energies predicted for the $11 \mathrm{a}^{\prime}(\mathbf{3}), 10 \mathrm{a}^{\prime}(4)$ and $6 \mathrm{a}^{\prime \prime}(5)$ orbitals is dependent upon the employed model of ionization and basis set, the global energy spreading for the three associated one-electron lines is merely insensitive to the employed theoretical level and is of the order of $0.2 \mathrm{eV}$. According to our simulations, contributions from the four one-electron ionization lines related to $7 \mathrm{a}^{\prime \prime}, 11 \mathrm{a}^{\prime}, 10 \mathrm{a}^{\prime}$ and $6 \mathrm{a}^{\prime \prime}$ orbitals $\mathbf{( 2 - 5 )}$ combine to form a sharp band around $\sim 12.24 \mathrm{eV}$, in agreement with the photoelectron spectra which exhibit a broad band extending from $11.5 \mathrm{eV}$ to $13 \mathrm{eV}$. A weak but discernable shoulder at $11.6 \mathrm{eV}$ in the experimental measurement is to ascribe to the $7 \mathrm{a}^{\prime \prime}(\mathbf{2})$ one-particle orbital. The calculations predict that the major components due to the $9 \mathrm{a}^{\prime}(6)$ and the $5 \mathrm{a}^{\prime \prime}(7)$ orbitals are separated by $\sim 0.6 \mathrm{eV}$. The two corresponding maxima locate at 13.27 and $13.86 \mathrm{eV}$ in the simulated ionization spectra, respectively. It seems thus reasonable to associate the $9 \mathrm{a}^{\prime}(\mathbf{6})$ component with a maximum at $13.1 \mathrm{eV}$ in the experimental spectrum, whereas the shoulder around $14.0 \mathrm{eV}$ seems to correlate with the 5a" (7) one-electron contribution.

In agreement with early but very limited Hartree-Fock calculations employing the 4-31G basis set [62b], the band at $15.8 \mathrm{eV}$ is assigned at the $\mathrm{ADC}(3)$ level to a one-electron state 
corresponding to the $8 \mathrm{a}^{\prime}(\mathbf{8})$ molecular orbital. Through space and bonding interactions (Fig. 2) associated with $\sigma-$ (or hyper) conjugation between optimally oriented methylenic $\left(\mathrm{CH}_{2}\right)$ groups [90] helps to substantially stabilize the $8 \mathrm{a}^{\prime}(\mathbf{8})$ orbital, by more than $2.1 \mathrm{eV}$ relative to the anti-bonding combination of $\mathrm{C}_{2 \mathrm{p}}$ atomic functions in the $5 \mathrm{a}^{\prime \prime}$ (7) orbital. An other interesting feature within the valence He I and He II photoelectron spectra of cyclopentene concerns the weak structure observed around $16.5 \mathrm{eV}$, which, by comparison with the simulated spectra, can be ascribed to two shake-up states of approximately equal strength $(\Gamma \sim 0.36)$ associated to the $7 \mathrm{a}^{\prime}$ (9) orbital, through the interplay of the $5 \mathrm{a}^{\prime \prime-1} 9 \mathrm{a}^{\prime^{-1}} 13 \mathrm{a}^{\prime \prime 1}\left((\mathrm{HOMO}-6)^{-1} \text { (HOMO }-5\right)^{-1}$ $\left.(\mathrm{LUMO}+13)^{+1}\right)$ electronic configuration, which locates the shake-up onset in the outer-valence region at $16.5 \mathrm{eV}$. Both at the $\mathrm{ADC}(3) / \mathrm{cc}-\mathrm{pVDZ}$ and $\mathrm{ADC}(3) / \mathrm{DZP}+$ levels, the calculations also predict for this orbital a third and weaker satellite line at $17.3 \mathrm{eV}(\Gamma=0.12)$ associated to the $4 \mathrm{a}^{\prime \prime-1}$ $9 \mathrm{a}^{\prime^{-1}} 13 \mathrm{a}^{\prime+1}\left((\mathrm{HOMO}-7)^{-1}(\mathrm{HOMO}-5)^{-1}(\mathrm{LUMO}+13)^{+1}\right)$ electronic configuration

Analysis of the LCAO eigenvectors indicates that the two orbitals [4a" (10), 6a' (11)] at the top of the inner-valence region are dominated by $\mathrm{C}_{2 \mathrm{~s}}$ contributions with some admixture of $\mathrm{C}_{2 \mathrm{p}}$ and $\mathrm{H}_{1 \mathrm{~s}}$ contributions. The two low-lying innermost orbitals $3 \mathrm{a}^{\prime \prime}$ (12) and $5 \mathrm{a}^{\prime}$ (13) exhibit through-space anti-bonding interactions and derive merely from contributions of $\mathrm{C}_{2 \mathrm{~s}}$ atomic functions. In contrast, the innermost orbital 4a' (14) which give rise to the broad shake-up bands between 25 and $28 \mathrm{eV}$ in the $\mathrm{ADC}(3)$ spectra (Fig.3, Table 2) is the result of an entirely bonding combination of $\mathrm{C}_{2 \mathrm{~s}}$ atomic functions. For the $4 \mathrm{a}^{\prime \prime}(\mathbf{1 0})$ and $6 \mathrm{a}^{\prime}(\mathbf{1 1})$ levels, the orbital picture of ionization appears to remain valid to some extent, whereas the orbital picture of ionization breaks down entirely for the innermost 3a" (12), 5a' (13), and 4a' (14) orbitals. Indeed, above 21 $\mathrm{eV}$, all the $\mathrm{ADC}(3)$ results predict that the ionization intensity resulting from the inner valence 
$3 a^{\prime \prime}(\mathbf{1 2}), 5 a^{\prime}(\mathbf{1 3}), 4 a^{\prime}(\mathbf{1 4})$ orbitals will severely split into many lines of individually very limited intensities, because of final state configuration interaction effects. Whatever the basis set, the theoretical predictions indicate that the most intense 4a" (10) and 6a' (11) ionization lines exhibit a pole strength larger than 0.6 in the energy region comprised between 17 and $20 \mathrm{eV}$ and are located at $\sim 17.6$ and $\sim 19.2 \mathrm{eV}$, respectively, which is in good agreement with the corresponding experimental values $(17.0$ and $19.0 \mathrm{eV})$ that can be inferred from the He II spectrum by Wiberg [62b]. Within this energy region, the reported ADC(3)/DZP+ calculations predict that the total spectroscopic strength which can be recovered through summation on all identified cationic states for the $3 \mathrm{a}^{\prime \prime}(\mathbf{1 2})$ and $5 \mathrm{a}^{\prime}(\mathbf{1 3})$ orbitals are equal to 0.707 and 0.503 , respectively, whereas the pole strength of the most intense line is of the order of 0.3 and 0.2 , respectively. Thus, the last peak that is seen in the He II PES measurements near $22 \mathrm{eV}$ can be reliably assigned to a complex satellite band derived from both the $3 \mathrm{a}^{\prime \prime}(\mathbf{1 2})$ and $5 \mathrm{a}^{\prime}(\mathbf{1 3})$ orbitals.

The simulated ADC(3) spectra indicate an enhanced shake-up splitting for the band associated to the $4 \mathrm{a}^{\prime}(\mathbf{1 4})$ orbital, the ionization of which leads to a multitude of states having extremely low pole strengths $(\Gamma<0.05)$. One further broad feature at higher electron binding energies emerge therefore in the energy region ranging from 25 to $28 \mathrm{eV}$ in the theoretical ADC(3) spectrum, in agreement with the EMS measurements (see Fig. 4). The total fraction of ionization intensity that is recovered at the ADC(3)/cc-pVDZ and ADC(3)/DZP+ levels from the $4 \mathrm{a}^{\prime}$ orbital is equal to 0.582 and 0.423 , respectively. A shoulder on the high-energy side of the last band (VII) observed around $25.85 \mathrm{eV}$ is seen in all the experimental (e, 2e) spectra, together with less pronounced structures embedded within a long correlation tail, extending from $\sim 28$ to 
$\sim 34 \mathrm{eV}$ [see Fig. 4]. In straightforward analogy with an exhaustive ADC(3) study [91] of the innermost bands of $n$-alkanes using the band-Lanczos diagonalization approach for diagonalizing the associated secular matrices, these structures and tail are most certainly ascribable to a quasi-continuum of shake-up and shake-off transitions with particularly small intensities $(\Gamma<0.005)$. Indeed, according to a comparison of energies obtained from single-point $\operatorname{CCSD}(\mathrm{T}) /$ aug-cc-pVTZ calculations upon the neutral and dication, the vertical double ionization threshold of cyclopentene in its puckered conformation lies at $\sim 25.6 \mathrm{eV}$. All the $2 \mathrm{~h}-1 \mathrm{p}$ shake-up states identified above that threshold are thus intrinsically very much dependent on the basis set and should rather be regarded as approximations to unbound states that lie in the continuum, and which are thus subject to decay via emission of a second electron. The shake-up fragmentation is further enhanced in this case because of the very low symmetry $\left(\mathrm{C}_{\mathrm{s}}\right)$ of cyclopentene, enabling many configuration interactions in the cation.

Overall, it is clear that the expected accuracy for the $\mathrm{ADC}(3)$ calculations is within $\sim 0.3 \mathrm{eV}$, which a comparison with the experimental one-electron binding energies shown in Table 2 confirms, except for orbital 7a"(2) ( $\sim 0.4 \mathrm{eV}$ discrepancy). Similar discrepancies were found for the $7 a^{\prime \prime}$ orbital of the norbornene cage compound [92] and the $3 b_{1}$ level of the thiophene cyclic compound [93]. The slightly larger discrepancies presumably may be indicative of strong geometrical relaxation effects and vibrational complications in a five-membered unsaturated ring which is subject to rather significant cyclic strains. At the 1p-GF/ADC(3) level, the impact of diffuse functions on the one-hole ionization energies is, as usual, very limited. Specifically, including such functions in the cc-pVDZ basis set results most generally in shifts of the one-electron ionization energies by less than $0.04 \mathrm{eV}$ towards higher electron binding energies 
(Table 2).

Inspection of Table 2 shows that the calculated pole strengths remain almost constant $\left(\Gamma_{n} \sim\right.$ $0.90 \pm 0.01)$ within the outer-valence region $(10-16 \mathrm{eV})$, and indicates that ionization processes at binding energies comprised between 10 and $16 \mathrm{eV}$ are qualitatively correctly described by the removal of one electron from a specific molecular orbital. Therefore, results obtained using the outer-valence Green's function (OVGF) approach confirms the empirical rule [51k] that OVGF pole strengths smaller than 0.85 corroborate a breakdown of the orbital picture of ionization at the $\mathrm{ADC}(3)$ level.

Clearly, standard Kohn-Sham orbital energies can not give particularly good insights into valence ionization spectra. The B3LYP orbital energies typically underestimate the corresponding experimental values for the electron binding energies by 2.3 to $3.3 \mathrm{eV}$. Such discrepancies are most common with standard exchange-correlation (XC) functionals. It is known [94] that XC functionals, be they implemented using the local spin density (LSD) or the generalized gradient approximations (GDA), do not have the correct Coulombic asymptotic behavior $(-1 / r)$ in the large $r$ region. Also, the equivalent of Koopman's theorem in Density Functional Theory (Janak's theorem [95]) only strictly holds for the Highest Occupied Molecular Orbital (HOMO). Despite the so-called meta-Koopmans theorem [96] that relates Kohn-Sham orbital energies to relaxed ionization energies, we would like to remind, again, that the DFT formalism does not explicitly account for final-state configuration interactions leading to the dispersion of the ionization intensity into shake-up processes. At best, the DFT formalism can only implicitly account for electronic correlation and relaxation effects, within the framework of a one-electron (or quasi-particle) picture of ionization, through a mapping of 
Kohn-Sham orbitals onto Dyson orbitals. The B3LYP functional was certainly not designed to ensure such a mapping. Nonetheless, this functional has been shown to enable in general extremely good (but empirical) descriptions of the experimental electron momentum distributions inferred from EMS experiments. Specifically, in most EMS investigations, KS orbitals have been implicitly regarded as very adequate approximations to normalized Dyson orbitals. Nevertheless, the success of KS momentum distributions in EMS is most certainly the outcome of error cancellations (neglect of final-state correlation, i.e., relaxation effects on orbitals versus the too rapid falloff of the DFT exchange correlations potential at large distances due to the self-interaction error), an observation that is worth many assessments against the results of more reliable many-body calculations (see further section). Note that, in contrast with KS orbitals, application of Koopmans' theorem to the HF orbital energies leads to overestimations of the experimental one-electron ionization energies by 1.0 to $2.1 \mathrm{eV}$, due to the neglect of electron relaxation and correlation effects.

\section{Momentum distribution}

Prior to proceeding to detailed calculations of electron momentum distributions, it is worth considering ionization spectra obtained through EMS experiments upon cyclopentene. The valence shell binding energy spectra (BES) of cyclopentene in the range 2-34 eV recorded by Ren et al [47] at azimuthal angles equal to $\phi=1^{\circ}$ and $\phi=8^{\circ}$ are shown in Figures $4 \mathrm{a}$ and $\mathrm{b}$, respectively. The electron density distributions for the complete valence shell of cyclopentene $\left(\mathrm{C}_{5} \mathrm{H}_{8}\right)$ and the corresponding electron binding energy spectra were measured at an electron impact energy of $1200 \mathrm{eV}$ plus electron binding energy, using an energy dispersive multichannel (e, 2e) electron momentum spectrometer employing symmetric non-coplanar geometry. The 
series of individual Gaussian peaks that were obtained by Ren et al. through a leas-square fitting procedure for convolving and analyzing accordingly their (e, 2e) measurements is also reproduced in these figures. In Fig. 4, Gaussian peaks fitted to the individual electronic transitions are given in the form of dashed lines while their sum is represented by a solid line. The employed energy values for locating these Gaussian peaks were inferred from the available PES measurements [62]. The widths of Gaussian peaks account both for the EMS instrumental energy resolution and the Frank-Condon widths of the corresponding bands, again according to the PES records $[62 \mathrm{~b}, 96]$. Small adjustments in the position of bands have been made to compensate the asymmetries of the shapes of the Frank-Condon profiles.

It is now well-established that molecular orbitals with a p-type profile have vanishing (e, 2e) ionization cross sections at low electron momenta, i.e., when $\phi=0^{\circ}$. In contrast, these orbitals contribute significantly to the (e, 2e) ionization intensities at larger values of $\phi$, in particular, to those values that correspond to electron momenta ranging from $\sim 0.5$ to $\sim 1.0$ a.u. For example, see the momentum distribution associated to band I (Figure 5a). Conversely, a s-type orbital yields typically a maximal electron momentum density at $p \sim 0$ a.u. $\left(\phi=0^{\circ}\right)$, which decays exponentially with increasing electron momenta. The contribution of these orbitals therefore dominates in the ionization spectrum recorded at $\phi=0^{\circ}$, and tends to vanish from the spectra recorded at larger azimuthal angles.

In Ren's work [47], seven structures (I - VII) could be identified in the electron binding energy spectrum of Fig. 4, among which four bands (I - IV) that could be reliably resolved in the outer-valence energy region. The vertical ionization threshold of cyclopentene relates to the HOMO [12a' (1)] and is located at $9.15 \mathrm{eV}$. The three other outer-valence bands are 
experimentally found at $11.95,14.19$ and $16.05 \mathrm{eV}$. The first band in the inner-valence region is seen at $18.30 \mathrm{eV}$ and incorporates the main lines and shake-up satellites associated to the $4 \mathrm{a}^{\prime}(\mathbf{1 0})$ and 6a" (11) orbitals. The deeper-lying $\mathrm{C}_{2 \mathrm{~s}}$ levels could not be individually resolved, but rather yield broad signals in the experimental spectra, due to the severity of the shake-up fragmentation. The new $\mathrm{ADC}(3)$ calculations clearly indicate that the band observed at $\sim 22.3 \mathrm{eV}$ in the EMS measurements by Ren et al [47] relates to a complex set of shake-up lines originating from the $3 \mathrm{a}^{\prime \prime}$ and $5 \mathrm{a}^{\prime}$ inner-valence $\left(\mathrm{C}_{2 \mathrm{~s}}\right)$ orbitals, whereas the very broad and intense band at binding energies comprised between 25 and $34 \mathrm{eV}$ is ascribed to shake-off states associated to ionization of the innermost $4 \mathrm{a}^{\prime}$ valence level.

In order to compare the experimentally inferred electron momentum distribution for bands I - VII with spherically averaged electron momentum distributions derived from all the 1p-GF/ADC(3) and DFT/B3LYP calculations, we propose to pursue the discussion of the one-electron (e, 2e) ionization bands of cyclopentene through simulations of electron momentum distributions for the $\left\{12 \mathrm{a}^{\prime \prime}(\mathbf{1})\right\},\left\{7 \mathrm{a}^{\prime \prime}+10 \mathrm{a}^{\prime}+11 \mathrm{a}^{\prime}+6 \mathrm{a}^{\prime \prime}+9 \mathrm{a}^{\prime}(\mathbf{2}-\mathbf{6})\right\},\left\{5 \mathrm{a}^{\prime \prime}(7)\right\}$ and $\left\{8 \mathrm{a}^{\prime}+7 \mathrm{a}^{\prime}(\mathbf{8 , 9})\right\}$ sets of orbitals, ascribed to bands I, II, III and IV at 9.2, 12.0, 14.2 and $16.1 \mathrm{eV}$, respectively (Table 2). For the latter orbital [7a' (9)], the retained fraction of ionization intensity that contributes to band IV was set equal to $\Gamma_{\text {tot }}=0.374+0.361=0.735$. Running further toward the more challenging inner-valence region, we propose the following assignment for the (e, 2e) ionization bands at 18.30, 22.30 and $25.85 \mathrm{eV}$ : band $(\mathbf{V})=7 \mathrm{a}^{\prime}(\mathbf{9})[0.123]+4 \mathrm{a}^{\prime \prime}(\mathbf{1 0})\left[\Gamma_{\text {tot }}=0.796\right]$ $+6 \mathrm{a}^{\prime}(\mathbf{1 1})\left[\Gamma_{\text {tot }}=0.872\right]$; band $(\mathbf{V I})=3 \mathrm{a}^{\prime \prime}(\mathbf{1 2})\left[\Gamma_{\mathrm{tot}}=0.707\right]+5 \mathrm{a}^{\prime}(\mathbf{1 3})\left[\Gamma_{\mathrm{tot}}=0.503\right]$; and band VII $=$ $5 \mathrm{a}^{\prime}(\mathbf{1 3})\left[\Gamma_{\text {tot }}=0.186\right]+4 \mathrm{a}^{\prime}(\mathbf{1 4})\left[\Gamma_{\text {tot }}=0.395\right]$. These bands are thus correspondingly ascribed to shake-up lines arising from the $4 a^{\prime \prime}, 6 a^{\prime}, 3 a^{\prime \prime}, 5 a^{\prime}, 4 a^{\prime}(\mathbf{1 0}-\mathbf{1 4})$ orbitals (see Table 2$) . \Gamma_{\text {tot }}$ denotes 
here the total fraction of ionization intensity that could be recovered for the orbital of interest, through a summation of the pole strengths of all identified lines within the energy range of interest.

Spherically averaged $\mathrm{ADC}(3)$ momentum distribution profiles have been calculated for each resolvable band in the angular-dependent EMS ionization spectra by summing the momentum distributions recovered from the associated un-normalized Dyson orbitals for each identified shake-up line within the corresponding electron binding range. To fit experimental data and enable consistent comparisons with momentum distributions recovered from un-normalized Dyson orbitals, all the momentum distributions generated from KS orbitals have been correspondingly scaled by a factor equal to the above cited total pole strengths $\Gamma_{\text {tot. }}$ The momentum-space orbital densities experimentally inferred from the angular dependences of the recorded $(e, 2 e)$ intensities for the seven identified ionization valence bands in Figure 4 are therefore compared in Figures 5-11 with the theoretical profiles generated at various theoretical levels from 1p-GF/ADC(3) and DFT calculations, using the band partition that has been proposed above on the ground of our $\mathrm{ADC}(3) / \mathrm{DZP}+$ calculations. In these figures, we also provide spherically averaged momentum distributions obtained at the same theoretical levels from the individual orbitals. These momentum distributions account for a finite angular experimental resolution $\left(\Delta \theta= \pm 0.6^{\circ}\right.$ and $\left.\Delta \phi= \pm 1.2^{\circ}\right)$, which implies a momentum resolution of 0.1 a.u. (one standard deviation) at an electron impact energy of $1.2 \mathrm{keV}$.

The experimental and theoretical momentum distributions for the $12 \mathrm{a}^{\prime}$ (1) orbital defining the HOMO level of cyclopentene are compared in Fig. 5. This orbital obviously dominantly relates to the $\pi$-chemical bond of cyclopentene (Figure 2), and its p-type momentum distribution 
therefore nicely reflects the presence of one nodal surface (Figure 2) that almost exactly follows the plane defined by the double bond and the adjacent single bonds. Upon comparing theory with experiment, it is clear that diffuse functions are essential for quantitatively reproducing the location of the maximum in the experimental profile, at $p_{\mathrm{MAX}} \sim 0.65$ a.u. In other words, this rather limited $p_{\mathrm{MAX}}$ value characterizing the $12 \mathrm{a}^{\prime}(\mathbf{1})$ momentum distribution profile images the rather diffuse nature of this orbital in configuration $(r)$ space. Very obviously, the B3LYP/aug-cc-pVTZ Kohn-Sham orbital momentum distribution for the 12a' (1) orbital leads to a rather pronounced underestimation of the $(\mathrm{e}, 2 \mathrm{e})$ cross sections around 0.75 a.u. In contrast, the agreement between the experimental and $\mathrm{ADC}(3) / \mathrm{DZP}+$ Dyson orbital momentum distribution for this level is almost perfect, except for a slightly more pronounced turn-up of the (e, 2e) ionization intensities at low electron momenta, which may be indicative of a partial breakdown of the plane wave impulse approximation [98]. Such a breakdown is likely to occur, considering the presence in this orbital of a second nodal surface perpendicular to the ring, and the resemblence of the 12a' (1) orbital with a d-type atomic orbital (Figure 2). Clearly therefore, diffuse functions and benchmark many-body treatments are here needed for accurately reproducing the experimental $(\mathrm{e}, 2 \mathrm{e})$ electronic density distribution associated to the HOMO in momentum space.

The outer-valence (e, 2e) ionization band II observed at $11.95 \mathrm{eV}$ encompasses the $7 \mathrm{a}^{\prime \prime}$ (2), $11 a^{\prime}(3), 10 a^{\prime}(4), 6 a^{\prime \prime}(5)$ and $9 a^{\prime}(6)$ set of orbitals. The $7 a^{\prime \prime}(2)$ orbital belongs to the $\sigma$-band system but yields a p-type momentum distribution (Fig. 6a). This is in line with the presence of an odd number of nodal surfaces and thus, with the antisymmetric nature of this orbital within the $\mathrm{C}_{\mathrm{s}}$ point group of the molecule. Note in particular that this orbital merely localizes and 
emphasizes therefore anti-bonding $\mathrm{C}-\mathrm{C}$ contributions in the puckered plane, i.e. the $\mathrm{C}_{3} \mathrm{C}_{4} \mathrm{C}_{5}$ plane (Fig.1). The more strongly localized nature of this orbital yields very naturally therefore a larger $p_{\text {MAX }}$ value ( $\sim 1.0$ a.u.). All theoretical predictions, whatever the employed model and basis set, provide the expected p-type profile. Note that KS orbitals yield here vanishing (e, 2e) ionization intensities at small electron momenta, whereas the $(\mathrm{e}, 2 \mathrm{e})$ intensities calculated at these momenta from our Dyson orbitals remain still rather significant. This difference rather clearly reflects the shortcomings, i.e. the too fast decay, of the B3LYP electronic potential in the asymptotic region.

The orbital 11a' (3) dominantly relates to $\sigma_{\mathrm{C}-\mathrm{H}}$ bond contributions associated with the $\mathrm{CH}_{2}$ group that lies opposite to the double bond (Figure 2). These $\sigma_{\mathrm{C}-\mathrm{H}}$ bond contributions exhibit in turn an out-of-phase relationship. The p-type profile which characterizes the $11 a^{\prime}(3)$ momentum distribution (Fig. 6b) images therefore the anti-bonding nature of this orbital, and more specifically the nodal surface that approximately follows the puckered-plane (Fig. 2). The rather low $p_{\operatorname{MAX}}$ value $(\sim 0.83$ a.u.) for this profile reflects the more diffuse nature of the peripheral C-H electron densities, compared with the C-C ones. Again, compared with its B3LYP Kohn-Sham counterpart, the 11a (3) Dyson orbital momentum distribution yields much larger (e, 2e) ionization cross sections at the origin of momentum space cross around momentum origin (Fig. 6b), which again reflects the shortcomings of standard exchange-correlation functionals in the asymptotic region $(r \rightarrow \infty)$. We also find that the B3LYP/DZP+ and B3LYP/aug-cc-pVTZ momentum distributions are almost identical, which makes us believe that the momentum distributions associated to the $\mathrm{ADC}(3) / \mathrm{DZP}+$ Dyson orbitals should also be close enough to convergence with respect to further improvements of the basis set. 
The more complex momentum distribution displayed in Fig. 6c relates to an orbital 10a' (4) characterized by the presence of three parallel nodal surfaces across the five-membered ring and multiple $\sigma_{\mathrm{C}-\mathrm{C}}$ and $\sigma_{\mathrm{C}-\mathrm{H}}$ bonding interactions therefore (Figure 2). It is thus rather logical to observe within this electron momentum distribution two maxima of the electron density at $p=$ $\sim 0.3$ a.u. and at $p=\sim 1.1$ a.u., along with two minima at 0.0 and $\sim 0.7$ a.u. (Fig.6c). All theoretical results qualitatively agree on the shape of the $10 \mathrm{a}^{\prime}$ electron momentum distribution profile. Again, the momentum densities at low momenta ( 0.0 to 0.7 a.u.) are found to be particularly sensitive to the employed basis sets and models of ionization.

The EMS momentum distribution related to orbital 6a" (5) (Fig. 6d) is also a doublep-type profile characterized by two main components at $p \sim 0.33$ a.u. and at $p \sim 1.2$ a.u (Fig. $6 \mathrm{~d}$ ). Again, the presence of two maxima in the momentum distributions and the enhancement of the electron densities at large electron momenta reflects the presence of three parallel nodal surfaces across the ring. Compared with the 10a' momentum distribution of Fig. 6c, the stronger depletion of the relative (e, 2e) ionization intensities at $p \sim 0.0$ a.u. in Fig. $6 \mathrm{~d}$ also images the antisymmetric nature of the $6 \mathrm{a}^{\prime \prime}(\mathbf{5})$ orbital with regards to the symmetry plane of cyclopentene in its $\mathrm{C}_{\mathrm{s}}$ conformation. The low momentum section is once more extremely sensitive to the incorporation of diffuse function in the basis set and to the employed treatment of electronic correlation and relaxation.

Similar observations can be made for the $9 \mathrm{a}^{\prime}$ (6) orbital (Fig. 6e), the momentum density of which gives rise to a strong contribution at $p \sim 0.37$ a.u. and to a secondary peak of less intensity at $p \sim 1.16$ a.u, in line with the presence of multiple nodal surfaces across the ring. Again, diffuse functions in the basis set and the correlation model appear to have a noticeable impact on the 
momentum distributions at low electron momenta, around 0.4 a.u.

Whatever the employed model, the total momentum distribution computed for this set of one-electron ionization lines orbitals exhibits also a double-peak distribution, which is qualitatively consistent with the experimental records. Compared with the global $\mathrm{ADC}(3) / \mathrm{cc}-\mathrm{pVDZ}$ or $\mathrm{ADC}(3) / \mathrm{DZP}+$ theoretical momentum distributions for band II (Fig. 6f), the B3LYP/aug-cc-pVTZ results provides a slightly superior description of the relative intensity and position of two of the maxima seen in the measured distribution. This slightly better agreement with experiment may somehow be artificial, since all calculations fail to reproduce a turn-up of (e, 2e) ionization intensities at 0.0 a.u., as well as a fourth peak in the momentum distributions at $p \sim 0.6$ a.u. These discrepancies may be due to a breakdown of the PWIA [orbital 6a" (5) bears indeed again strong similarities with a d-type orbital or a $\pi^{*}$-type orbital], or unusually fast geometrical distortions, and symmetry breaking therefore, due to a Jahn-Teller effect and vibronic interactions originating from the ionization of orbitals with very similar electron binding energies.

Going further towards the next spectral band III produced by ionization of an electron out of orbital 5a" (7), we observe an almost perfect agreement between the various theoretical predictions made for this level (Figure 7). The 5a" (7) orbital can be described as an anti-bonding combination of strongly localized $\pi\left(\mathrm{CH}_{2}\right)$ contributions, giving rise to a p-type electron momentum distribution. Localization of the $5 \mathrm{a}^{\prime \prime}$ (7) electron density in peripheral C-H bonds yields again a rather limited value $\left(p_{\mathrm{MAX}} \sim 0.73\right.$ a.u.) for the location of the maximum in the associated momentum density profile. Very significant discrepancies are nonetheless seen between all theoretical calculations and the experimental measurements at momenta below 0.3 
a.u. The analysis on the ground of Dyson orbitals corroborates therefore the conclusion [47] that the discrepancies between experimental data and theoretical calculations below 0.3 a.u. for the 5a" orbital are not due to a possible uncertainty in the curve fitting procedures, but more probably the outcome of strong distorted wave effects [98]. This observation is once more consistent with the fact that the 5a" (7) orbital of cyclopentene exhibits two perpendicular nodal surfaces, and can be described as a $\pi^{*}$-like or d-like orbital therefore [98]. New EMS measurements on cyclopentene at various impact energies for the impinging electron are clearly indeed, in order to further verify the origin of the extremely strong "turn up" of the (e, 2e) 5a" (7) cross sections in the low momentum region.

The band (IV) that experimentally emerges near $16.05 \mathrm{eV}$ is due to the outer-valence orbital doublet $8 \mathrm{a}^{\prime}(\mathbf{8})$ and $7 \mathrm{a}^{\prime}(\mathbf{9})$. All calculated (e, 2e) ionization intensities for this doublet give almost identical results (Figure 8). The global electron momentum profile is of the s-type, and reflects the unquestionable dominance of the (e, 2e) ionization intensities arising from the $7 \mathrm{a}^{\prime}(9)$ orbital. In excellent agreement with experiment, the theoretical simulations for this global profile also exhibit a shoulder at $\sim 0.8$ a.u., which can also be traced back to the $7 \mathrm{a}^{\prime}(9)$ orbital (compare Figures $8 \mathrm{~b}$ and $\mathrm{c}$ ). Indeed, this orbital lies at the bottom of the outer-valence region, and merely relates therefore to contributions from $\mathrm{C}_{2 \mathrm{p}}$ and $\mathrm{H}_{1 \mathrm{~s}}$ atomic orbitals associated to the $\mathrm{C}-\mathrm{H}$ bond, which overlap in an all-bonding way (Figure 2). This orbital is therefore subject to a substantial energy stabilization by virtue of through-space $\sigma$-conjugation effects (also referred to a methylenic hyperconjugation effects in $n$-alkanes $[90,99])$. The contribution from the peripheral $\mathrm{C}-\mathrm{H}$ crown of the molecule gives rise to particularly large electron densities at small values of $p$ in the momentum electron distribution associated to band IV. The symmetric 
characteristics and the strong delocalization of the 7a' (9) orbital onto the peripheral $\mathrm{C}-\mathrm{H}$ crown explain the importance of the peak at vanishing electron momenta in the associated distribution of momentum densities. By virtue of electron confinement in the configuration space, the presence of one closed nodal surface that approximately follows the carbon backbone (Figure 2) also explains the presence of a shallow minimum at $p \sim 0.6$ a.u and of a second minor maximum at $p \sim 0.89$ a.u. in the momentum distribution computed for the $7 \mathrm{a}^{\prime}(9)$ orbital (Figure $8 \mathrm{~b}$ ). This latter minimum almost coincides with the position of the maximum ( $p \sim 0.5$ a.u. $)$ in the p-type electron momentum distributions (Figure 8a) that have been computed for the $8 \mathrm{a}^{\prime}(\mathbf{8})$ orbital, and takes therefore the form of an inflection (Figure 8c) in the global momentum distribution inferred for band IV $\left(8 \mathrm{a}^{\prime}(\mathbf{8})+7 \mathrm{a}^{\prime}(\mathbf{9})\right)$. This rather low value for $p_{\max }$ is also indicative of a partial delocalization of the $8 \mathrm{a}^{\prime}(\mathbf{8})$ orbital due to $\sigma$-conjugation between the $\mathrm{C}_{\mathrm{sp}}{ }^{3}$ carbon atoms and their $\mathrm{H}$ substituents. All employed models yield here almost identical momentum distributions.

In view of the $\mathrm{ADC}(3)$ results, band $\mathbf{V}$ at $18.30 \mathrm{eV}$ in the EMS spectrum of cyclopentene is attributed to the main ionization lines and a few satellite lines with limited pole strength $\Gamma$ (most of them smaller than 0.1 ) that relate to the highest-lying orbitals within the inner-valence region, namely the 4a" (10) and 6a' (11) orbitals. These orbitals exhibit both two nodal surfaces across the ring (Figure 2) and their momentum distributions are both of the p-type (Figures 9a and b). The (e, 2e) ionization intensity of the $4 \mathrm{a}^{\prime \prime}(\mathbf{1 0})$ orbital strictly vanishes at $p \sim 0$ (Fig. 9a), in line with the antisymmetry of the orbital with regards to the mirror plane of the molecule, whereas the $6 \mathrm{a}^{\prime}(\mathbf{1 1})$ orbital produces minimal but non-vanishing $(\mathrm{e}, 2 \mathrm{e})$ ionization intensities at $p \sim 0$ a.u (Fig. 9b). Upon examining Figure 9, it is clear that the differences between the various models 
become almost insignificant when entering the inner-valence region. Compared with experiment, all calculations correctly predict a peak at $p=0.74$ a.u. The calculations fail again to quantitatively reproduce a very significant turn-up of the $(e, 2 e)$ ionization intensity at low momenta values ( $p<0.3$ a.u.), a feature that may once more be indicative of a breakdown of the plane wave impulse approximation for these two levels, an interpretation to relate with the d-like topologies of the $4 \mathrm{a}^{\prime \prime}(\mathbf{1 0})$ and $6 \mathrm{a}^{\prime}(\mathbf{1 1})$ orbitals, which both exhibit two perpendicular nodal surfaces (Figure 2). Note nonetheless that including within band $\mathbf{V}$ the contribution of a rather intense shake-up line $[\Gamma=0.123]$ at $17.28 \mathrm{eV}$ that finds its origin into ionization of the $7 \mathrm{a}^{\prime}$ (9) orbital helps to considerably improves the quality of the prediction made for the electron distribution recovered for band $\mathbf{V}$ : upon including this satellite, we can indeed predict a slight turn-up of the (e, 2e) ionization intensities (Fig. 9c). In other words, the experimental momentum distributions qualitatively corroborate the presence of a $\sigma^{-2} \sigma^{*^{+1}}$ satellite in this energy region.

Band VI falls near $22.30 \mathrm{eV}$, thus in the middle of the inner valence region of cyclopentene. Upon examining Table 2 and Figure 3, it is clear that this band relates to a complex set of shake-up lines originating from the $3 \mathrm{a}^{\prime \prime}(\mathbf{1 2})$ and 5a' (13) orbitals. The p-type orbital momentum distributions characterizing these orbitals clearly reflect the presence of one unique nodal surface (Figure 2). Again, the B3LYP Kohn-Sham orbitals and the ADC(3)/DZP+ Dyson orbitals lead to almost equal momentum distributions. The results of our calculations for band VI are compared with experimental records in Fig. 10c. In this case, the best match with regards to the position of the maximum $(0.5$ a.u. $)$ and with the overall shape of the profile is ensured with the $\mathrm{ADC}(3) / \mathrm{DZP}+$ results. It is also rather clear that the inclusion of diffuse functions in the 
cc-pVDZ basis sets has almost no impact on the angular dependence of the (e, 2e) ionization intensities for the KS orbitals associated to these levels.

The last energy band (VII), which is located at $25.85 \mathrm{eV}$ in the EMS ionization spectra (Fig.4), is dominated by a large number of satellites associated with ionization of the two innermost valence orbitals, namely $5 \mathrm{a}^{\prime}(\mathbf{1 3})$ and $4 \mathrm{a}^{\prime}(\mathbf{1 4})$. Fig. 11a shows that the innermost orbital 4a' has a "s-type" momentum distribution (Fig. 11b), which is in line with the all bonding nature of this molecular orbital (Fig. 2). Among all presented computations, the best results in predicting the shape of the experimental profile are again obtained with the ADC(3)/DPZ+ Dyson orbital.

Despite a very likely loss of ionization intensity in further unidentified shake-up processes that should contribute to a correlation tail at much higher electron binding energies [91], it is nonetheless clear that the B3LYP Kohn-Sham orbitals and the ADC(3) Dyson orbitals lead to qualitatively very similar momentum distributions for bands VI and VII, an observation which corroborates the idea that shake-up states "borrow" their intensity to specific one-electron levels. Diffuse functions have once more no significant influence on the computed electron momentum distributions, which reflects the inherently more strongly localized nature of inner-valence levels.

\section{Conclusions}

A thorough theoretical study of the electronic structure and related Dyson orbital momentum distributions of cyclopentene has been presented, in order to exhaustively interpret available experimental data obtained using Electron Momentum Spectroscopy. The latter technique is a powerful approach that combines electron-impact ionization and electron 
scattering, and enables therefore detailed experimental reconstructions of electron densities associated to specific ionization channels, i.e. orbital densities. As with any ionization experiment, the analysis of the angular dependence of the measured cross sections is nonetheless subject to many complications, like electronic relaxation and correlation effects, shake-up and shake-off processes, vibronic coupling interactions or distorted wave effects.

To carry out reliable enough analyses of such experiments, we first reassigned the available (e, 2e) and photo- (He I and He II) ionization spectra on the ground of thorough Green's function calculations of one-electron and shake-up valence ionization energies, in conjunction with the third-order algebraic diagrammatic construction $[\mathrm{ADC}(3)]$ scheme. These calculations enable insight with one-electron binding energies within an accuracy of a few tenths of an eV, and indicate that the orbital picture of ionization breaks down at binding energies larger than $\sim 17 \mathrm{eV}$. Electron momentum distributions that were experimentally inferred from the angular dependence of the (e, 2e) ionization intensities in EMS experiments at an electron impact energy of $1200 \mathrm{eV}$ were then analyzed on the basis of a band partitioning which is consistent with the computed $\mathrm{ADC}(3)$ ionization spectra. In this analysis, we resorted to a comparison of the experimental results with spherically averaged (e, 2e) electron momentum distributions that were computed for each identified ionization channels or resolvable bands using an adaptation of the MOMAP methodology by Brion et al [76] for Fourier Transforming to momentum space and spherically averaging the related Dyson orbitals, taking into account the finite angular resolution of the $(\mathrm{e}, 2 \mathrm{e})$ spectrometers for convolving the momentum distributions. This analysis enabled us to considerably improve, both from quantitative and qualitative viewpoints, our understanding of the electronic structure and related excitation properties of cyclopentene, and 
the influence of electronic correlation and relaxation effects on the apparent shape of orbitals.

The experimental momentum distributions were therefore found to faithfully reflect the spread and topology of the underlying molecular orbitals. More specifically, these momentum distributions have enabled us to demonstrate the rather strongly localized nature of the electron level associated to the unique $\pi$-bond in the molecule, and conversely identify from the apparent experimental distributions very specific fingerprints, at the bottom $(16.6 \mathrm{eV})$ of the outer-valence region, for $\sigma$-conjugation effects and delocalization of the associated ( $\left.7 \mathrm{a}^{\prime}\right)$ electron density onto the peripheral $\mathrm{C}-\mathrm{H}$ crown. This analysis also confirms the finding that the latter outer-valence orbital gives rise to a satellite at $17.3 \mathrm{eV}$ that has sufficient strength $(\Gamma=0.13)$ for producing sizable enough fingerprints in the electron momentum distributions recorded at the top $(17.3 \mathrm{eV})$ of the inner-valence region. In line with the rather low symmetry point group $\left(\mathrm{C}_{\mathrm{s}}\right)$ of cyclopentene in its ground state geometry, which enables many interactions between excited electronic correlations in the cation, very significant differences have been noted for the five outermost one-electron ionization lines between the momentum distributions computed from ADC(3) Dyson orbitals and B3LYP Kohn-Sham orbitals. These discrepancies and a rather strong dependence towards the presence of diffuse functions in the basis set rather clearly reflect therefore well-known shortcomings of this functional in the asymptotic region [511, 51o and references therein]. The $\mathrm{ADC}(3)$ Dyson orbital momentum distributions still fail to reproduce very significant turn-ups of the $(e, 2 e)$ ionization intensities recovered from the $5 \mathrm{a}^{\prime \prime}$ and $4 \mathrm{a}^{\prime \prime}+6 \mathrm{a}^{\prime}$ orbitals. In line with the d-like topology of the underlying orbitals, we interpret these discrepancies between theory and experiment as the outcome of a pronounced breakdown of the plane wave impulse approximation (i.e. distorted wave effects). 


\section{Acknowledgements}

This work has been supported by the National Natural Science Foundation of China under contract No. 10575062 and the Specialized Research Fund for the Doctoral Program of Higher Education under contract No. 20050003084. The authors also acknowledge the financial support by the Fonds voor Wetenschappelijk Onderzoek-Vlaanderen (FWO) and the Bijzonder OnderzoeksFonds (BOF) of the Hasselt University. Yanru Huang has been working nine months (15th June 2006 - 14th March 2007) as a PhD student in the group of Theoretical Chemistry at Hasselt University, thanks to a fellowship obtained within the framework of a bilateral program for scientific cooperation between Belgium (Flanders) and P.R. China. Michael S. Deleuze thanks all members of the Laboratory of Polarization Physics at Tsinghua University for their kind hospitality during his stay in Beijing in September 2007. 


\section{References}

[1] J. Laane and R. C. Lord, J. Chem. Phys., 1967, 47, 4941.

[2] L. A. Carriera, R. C. Lord and T. B. Top. Malloy, Curr. Chem., 1979, 82, 1.

[3] J. R. Villarreal, L. E. Bauman, J. Laane, Q. C. Harris and S. F. Bush, J. Chem. Phys., 1975, 63, 3727 .

[4] J. R. Villarreal, L. E. Bauman and J. Laane, Phys. Chem., 1976, 80, 1172.

[5] J. Lascombe, D. Cavagnat, J. C. Lassegues, C. Rafillipomanana and C. Biran, J. Mol. Struct., 1984, 113, 179.

[6] C. W. Beckett, N. K. Freeman and K. S. Pitzer, J. Am. Chem. Soc., 1948, 70, 4227.

[7] L. M. Sverdlov and E. N. Krainov, Opt. Spectrosc. (USSR), 1959, 6, 214.

[8] A. LeRoy and J. C. Thonvenou, C. R. Acad. Sci., 1967, B37, 545.

[9] T. Ueda and T. Shimanouchi, J. Chem. Phys., 1967, 47, 5018.

[10] W. D. Wertz, D. F. Bocian and M. J. Hazouri, Spectrochim. Acta, 1973, 29A, 1439.

[11] W. C. Harris and C. T. Longshore, J. Mol. Struct., 1973, 16, 187.

[12] J. R. Villarreal, J. Laane, S. F. Bush and W. C. Harris, Spectrochim. Acta, 1979, 35A, 331.

[13] W. H. Green, J. Chem. Phys., 1970, 52, 2156.

[14] M. Besnard, J.-C. Lassegues, Y. Guissani and J.-L. Leicknam, Mol. Phys., 1984, 53, 1145.

[15] J. R. Villarreal, Ph.D Thesis, Department of Chemistry, Texas A\&M University, 1976.

[16] J. Lascombe, D. Cavagnat, J. C. Lassegues, C. Rafillipomanana and C. J. Biran, Mol. Struct , 1984, 113, 179.

[17] C. Rafilipomanana, D. Cavagnat and J. C. Lassegues, J. Mol. Struct., 1985, 129, 215. 
[18] J. R. Durig and L. A. Carreira, J. Chem. Phys., 1972, 56, 4966.

[19] T. H. Chao and J. Laane, Chem. Phys. Lett., 1972, 14, 595.

[20] W. D.Wertz, D. F. Bocian and M. J. Hazouri, Spectrochim. Acta, 1973, 29A, 1439.

[21] W. C. Harris and C. T. Longshore, J. Mol. Struct., 1973, 16, 187.

[22] J. R. Villarreal, L. E. Bauman, J. Laane, Q. C. Harris and S. F. Bush, J. Chem. Phys., 1975, 63, 3727 .

[23] J. R. Villarreal, L. E. Bauman and J. Laane, J. Phys. Chem., 1976, 80, 1172.

[24] C. Rafilipomanana, D. Cavagnat, R. Cavagnat, J. C. Lassegues and C. Birat, J. Mol. Struct., 1985, 127, 283.

[25] G. W. Jr. Rathjens, J. Chem. Phys., 1962, 36, 2401.

[26] S. S. Butcher and C. C. Costain, J. Mol. Spectrosc., 1965, 15, 40.

[27] L. H. Acharpen, J. Chem. Phys., 1968, 48, 3552.

[28] M. I. Davis and T. W. Muecke, J. Phys. Chem., 1970, 74, 1104.

[29] T. B. Jr. Malloy, J. Mol, Spectrosc., 1972, 44, 504.

[30] T. B. Jr. Malloy and L. A. Carreira, J. hem. Phys., 1979, 71, 2488.

[31] L. E. Bauman, P. M. Killough, J. M. Cooke, J. R. Villarreal and J. Laane, J. Phys. Chem., $1982,86,2000$.

[32] T. L. Smithson, J. A. Duchkett, R. Paul, H. Wieser and F. W. Biress, Mol. Phys., 1984, 53, 1495.

[33] J. Laane, J. Mol. Struct., 1985, 126, 99.

[34] I. P. Hamilton, L .C. Light and K. B. Whaley, J. Chem. Phys., 1986, 85, 5151.

[35] R. Champion, P. D. Godfrey and F. L. Bettens, J. Mols. Spectrosc., 1991,147,488. 
[36] L. Sztraka, Acta Phys. Hung., 1988, 63, 143.

[37] L. Sztraka, J. Mol. Struct., 1990, 218, 327.

[38] J. H. Cho and L. Kleinman, Phys. Rev. B, 2001, 64, 235420.

[39] Y. Yamashita, K. Hamaguchi, S. Machida, K. Mukai, J. Yoshinobu, S. Tanaka and M. Kamada, Appl. Surf. Sci., 2001, 172, 169.

[40] G. P. Lopinski, D. D. M. Wayner and R. A. Wolkow, Nature, 2000, 406, 48.

[41] J. S. Hovis, H. Liu and R. J. Hamers, Surf. Sci., 1998, 1, 402.

[42] H. B. Liu and R. J. Hamers, Surf. Sci., 1998, 416, 354.

[43] H. B. Liu and R. J. Hamers, J. Am. Chem. Soc., 1997, 119, 7593.

[44] R. J. Hamers, J. S. Hovis, S. W. Lee, H. B. Liu and J. Shan, J. Phys. Chem. B, 1997, 101, 1489.

[45] S. W. Lee, J. S. Hovis, S. K. Coulter, R. J. Hamers and C. M. Greenlief, Surf. Sci., 2000, $462,6$.

[46] J. H. Cho and L. Kleinman, Phys. Rev. B, 2002, 65, 245407.

[47] X. G. Ren, C. G. Ning, S. F. Zhang, G. L. Su, B. Li, H. Zhou, F. Huang, G. Q. Li and J. K. Deng, Phys. Rev. A, 2005, 72, 052712.

[48] I. E. McCarthy and E. Weigold, Rep. Prog. Phys., 1991, 54, 789.

[49] M. A. Coplan, J. H. Moore and J. P. Doering, Rev. Mod. Phys., 1994, 66, 985.

[50] E. Weigold and I. E. McCarthy, Electron Momentum Spectroscopy, Kulwer Academic Plenum Publishers, New York, 1999.

[51] (a) L. S. Cederbaum, J. Phys. B, 1975, 8, 290; (b) L. S. Cederbaum, J. Chem. Phys., 1975, 62, 2160; (c) L. S. Cederbaum, W. Domcke, J. Schirmer, W. von Niessen, G. H. F. Diercksen and 
W. P. Kraemer, J. Chem. Phys., 1978, 69, 1591; (d) L. S. Cederbaum, W. Domcke, J. Schirmer and W. von Niessen, Adv. Chem. Phys., 1986, 65, 115; (e) M. S. Deleuze and L. S. Cederbaum, Int. J. Quantum Chem., 1997, 63, 465; (f) M. S. Deleuze, M. G. Giuffreda, J.-P. François and L. S. Cederbaum, J. Chem. Phys., 1999, 111, 5851; (g) M. S. Deleuze and L. S. Cederbaum, Adv. Quantum. Chem., 1999, 35, 77; (h) M. S. Deleuze, M. G. Giuffreda, J.-P. François and L. S. Cederbaum, J. Chem. Phys., 2000, 112, 5325; (i) M. S. Deleuze, M. G. Giuffreda and J.-P. François, J. Phys. Chem. A, 2002, 106, 5626; (j) M. S. Deleuze, A. B. Trofimov and L. S. Cederbaum, J. Chem. Phys., 2001, 115, 5859; (k) M. S. Deleuze, J. Chem. Phys., 2002, 116, 7012; (1) M. S. Deleuze, J. Phys. Chem. A, 2004, 108, 9244; (m) A. W. Potts, D. M. P. Holland, A. B. Trofimov, J. Schirmer, L. Karlsson and K. Siegbahn, J. Phys. B: At. Mol. Opt. Phys., 2003, 36, 3129; (n) N. Kishimoto, Y. Hagihara, K. Ohno, S. Knippenberg, J.-P. François and M. S. Deleuze, J. Phys. Chem. A, 2005, 109, 10535; (o) M. S. Deleuze, Chem. Phys., 2006, 329, 22; (p) A. W. Potts, D. Edvardson, L. Karlsson, D. M. P. Holland, M. A. MacDonald, M. A. Hayes, R. Maripuu, K. Siegbahn and W. von Niessen, Chem. Phys., 2000, 254, 385; (q) A. B. Trofimov, J. Schirmer, D. M. P. Holland, L. Karlsson, R. Maripuu, K. Siegbahn and A. W. Potts, Chem. Phys., 2001, 263, 167; (r) A. W. Potts, A. B. Trofimov, J. Schirmer, D. M. P. Holland and L. Karlsson, Chem. Phys., 2001, 271, 337; (s) A. B. Trofimov, J. Schirmer, D. M. P. Holland, A. W. Potts, L. Karlsson, R. Maripuu and K. Siegbahn, J. Phys. B, 2002, 35, 5051.

[52] (a) L. S. Cederbaum and W. Domcke, Adv. Chem. Phys., 1977, 36, 205; (b) Y. Öhrn and G. Born, Adv. Quantum Chem., 1981, 13, 1; (c) J. V. Ortiz, Computational Chemistry: Reviews of Current Trends, edited by J. Leszczynski (World Scientific, Singapore, 1997), Vol. 2, p. 1; (d) W. von Niessen, J. Schirmer and L. S.Cederbaum, Comput. Phys. Rep., 1984, 1, 57. 
[53] (a) J. Schirmer, L. S. Cederbaum and O. Walter, Phys. Rev. A, 1983, 28, 1237; (b) H.-G. Weikert, H.-D. Meyer, L. S. Cederbaum and F. Tarantelli, J. Chem. Phys., 1996, 104, 7122; (c) J. Schirmer and G. Angonoa, J. Chem. Phys., 1989, 91, 1754.

[54] M. S. Deleuze and L. S. Cederbaum, Proceedings of the second international conference on Polymer Solid Interfaces: from Model to Real Systems (ICPSI2: J. J. Pireaux, J. Delhalle and P. Rudolf, Namur, Belgium,1998).

[55] (a) D. M. P. Holland, L. Karlsson and W. von Niessen, J. Electron Spectrosc. Relat. Phenom. 2001, 113, 221; (b) Y. R. Huang, C. G. Ning, S. F. Zhang, K. Liu, Z. H. Luo, J. K. Deng and M. S. Deleuze, submitted.

[56] (a) M. S. Deleuze, L. S. Cederbaum, J. Chem. Phys., 1996, 105, 7583; (b) W. N. Pang, J. F. Gao, C. J. Ruan, R. C. Shang, A. B. Trofimov and M. S. Deleuze, J. Chem. Phys., 2000, 112, 8043; (c) M. S. Delezue, W. N. Pang, A. Salarm and R. C. Shang, J. Am. Chem. Soc., 2001, 123, 4049 .

[57] S. Knippenberg, J.-P. François and M. S. Deleuze, J. Comput. Chem., 2006, 27, 1703.

[58] M. S. Deleuze and S. Knippenberg, J. Chem. Phys., 2006, 125, 104309.

[59] C. G. Ning, X. G. Ren, J. K. Deng, G. L. Su, S. F. Zhang, S. Knippenberg and M. S. Deleuze, Chem. Phys. Lett., 2006, 421, 52.

[60] Y. R. Huang, S. Knippenberg, B. Hajgato, J.-P. François, J. K. Deng and M. S. Deleuze, J. Phys. Chem. A, 2007, 111, 5879.

[61] C. G. Ning, B. Hajgato, Y. R. Huang, S. F. Zhang, K. Liu, Z. H. Luo, S. Knippenberg, J. K. Deng and M. S. Deleuze, Chem. Phys., 2007, Doi:10.1016.

[62] (a) G. Bieri, F. Burger, E. Heilbronner and J. R. Maier, Helv. Chim. Acta, 1970, 60, 2213; 
(b) K. B. Wiberg, G. B. Ellison, J. J. Wendoloski, C. R. Brundle and N. A. Kuebler, J. Am. Chem. Soc., 1976, 98, 7179; (c) D. G. Streets, A. W. Potts, J. C. S. Faraday Trans. II, 1974, 70, 1505.

[63] V. G. Zakrzewski and W. von Niessen, J. Comput.Chem., 1993, 14, 13.

[64] (a) C. E. Brion, Int. J. Quantum Chem., 1986, 29, 1397; (b) I. E. McCarthy and E. Weigold, Rep. Prog. Phys., 1988, 51, 299.

[65] (a) B. T. Pickup, Chem. Phys., 1977, 19, 193; (b) R. Mc Weeny, B. T. Pickup, Rep. Progr. Phys., 1980, 43, 1065; (c) M. S. Deleuze, B. T. Pickup, J. Delhalle, J. Mol. Phys., 1994, 83, 655; (d) G. M. Seabra, I. G. Kaplan, V. G. Zakrzewski and J. V. Ortiz, J. Chem. Phys., 2004, 121, 4142.

[66] A. D. Becke, J. Chem. Phys., 1993, 98, 5648.

[67] C. Lee, W. Yang and R. G. Parr, Phys. Rev. B, 1988, 37, 785.

[68] D. Feller, J. Chem. Phys., 1992, 96, 6104.

[69] J. M. L. Martin, J. El-Yazal and J.-P. François, Mol. Phys., 1995, 86, 1437; see also W. Koch and M. C. A. Holthausen, A chemist's guide to density functional theory, $2^{\text {nd }}$ ed., (V. C. H. Wiley: Weinheim, Germany, 2001).

[70] M. J. Frisch et al, GAUSSIAN98, Revision A.7, Gaussian Inc., Pittsburgh, PA, 1998.

[71] M. W. Schmidt, K. K. Baldridge, J. H. Jensen, S. Koseki, M. S. Gordon, K. A. Nguyen, T. L. Windus and S. T. Elbert, QCPE Bull., 1990, 10, 52.

[72] (a) A. Ruhe, Math. Comput., 1979, 33, 680; (b) H.-D. Meyer and S. Pal, J. Chem. Phys., 1989, 91, 6195.

[73] (a) B. Liu, Numerical Algorithms in Chemistry, Algebraic Methods, LBL-8158; Lawrence Berkeley Laboratory: Berkeley, California; (b) F. Tarantelli, A. Sgamellotti, L. S. Cederbaum 
and J. Schirmer, J. Chem. Phys., 1987, 86, 2201.

[74] T. H. Jr. Dunning, J. Chem. Phys., 1989, 90, 1007.

[75] T. H. Jr. Dunning and P. J. Hay, Methods of Electronic Structure Theory; H. F. Schaefer, Ed.; Plenmu Press: New York, 1977; Vol.2.

[76] See various contributions to the original HEMS program as recorded by Bawagan [A. O. Bawagan, Ph. D. Thesis, University of British Columbia (UBC), 1987]. The HEMS (now known as MOMAP) program has been extensively revised and extended at UBC by N. M. Cann and G. Cooper.

[77] (a) R. J. Mathar, Int. J. Quantum Chem., 2002, 90, 227; (b) W. J. Lentz, Appl. Opt., 1976, $15,668$.

[78] P. Duffy, M. E. Casida, C. E. Brion and D. P. Chong, Chem. Phys., 1992, 159, 347.

[79] N. Nevins, K. Chen and N. L. Alliger, J. Comput. Chem., 1996, 17, 669.

[80] M. K. Leong, V. S. Mastryukov and J. E. Boggs, J. Mol. Struct., 1998, 445, 149.

[81] S. Saebø, F. R. Cordell and J. E. Boggs, J. Mol. Struct., 1983, 104, 221.

[82] M. T. Tribble and N. L. Allignger, Tetrahedron, 1972, 28, 2147.

[83] (a) S. Lifson and A. Warshel, J. Chem. Phys., 1968, 49,5116; (b) A. Warshel and S. Lifson, ibid, 1970, 53, 582.

[84] N. L. Allinger, J. Am. Chem. Soc., 1977, 99, 8127.

[85] W. D. Allen, A. G. Császár and D. A. Horner, J, Am.Chem. Soc., 1992, 114, 6834.

[86] N. I. Jr. Allinger, J. Chem. Phys., 1972, 94, 5734.

[87] O. Ermer and S. J. Lifson, J. Am. Chem. Soc., 1973, 95, 4142.

[88] R. L. Rosas, C. Cooper and J. Laane, J. Phys. Chem., 1990, 94, 1830. 
[89] S. P. Kwasniewski, L. Claes, J.-P. François and M. S. Deleuze, J. Chem. Phys., 2003, 118 7823.

[90] (a) M. S. Deleuze, J. P. Denis and B. T. Pickup, J. Phys. Chem., 1993, 97, 5115; (b) M. S. Deleuze, J. Delhalle, B. T. Pickup and S. Svensson, J. Am. Chem. Soc., 1994, 116, 10715; (c) M. S. Deleuze, J. Delhalle and B. T. Pickup, J. Phys. Chem., 1994, 98, 2382; (d) M. S. Deleuze, J. Delhalle, D. H. Mosely and J.-M. André, Physica Scripta, 1995, 51, 111.

[91] A. Golod, M. S. Deleuze and L. S. Cederbaum, J. Chem. Phys., 1999, 110, 6014.

[92] S. Knippenberg, K. L. Nixion, H. Mackenzie-Ross, M. J. Brugger, F. Wang, M. S. Deleuze, J.-P. François and D. A. Winkler, J. Phys. Chem. A, 2005, 109, 9324.

[93] Y. R. Huang, B. Hajgató, C. G. Ning, S. F. Zhang, K. Liu, Z. H. Luo, J. K. Deng, M. S. Deleuze "Study of the Valence Wavefunction of Thiophene with High Resolution Electron Momentum Spectroscopy and Advanced Dyson Orbital Theories", accepted for publication in J. Phys. Chem. A

[94] S. Hamel, P. Duffy, M. Casida and D. R. Salahub, J. Electron Spectrosc. Relat. Phenom., $2002, \mathbf{1 2 3}, 345$.

[95] J. F. Janak, Phys. Rev. B, 1978, 18, 7165.

[96] (a) O. V. Gritsenko and E. J. Baerends, J. Chem. Phys., 2002, 117, 9154; (b) O. V. Gritsenko, B. Braïda and E. J. Baerends, J. Chem. Phys., 2003, 110, 1937.

[97] K. Kimura, S. Katsumata, Y. Achiba, T. Yamazaki and S. Iwata, Handbook of HeI Photoelectron Spectra of Fundamental Organic Molecules, (Japan Scientific Society, Tokyo, 1981).

[98] (a) C. E. Brion, Y. Zheng, J. Rolke, J. J. Neville, I. E. McCarthy and J. Wang, J. Phys. B, 
1998, 31, L223; (b) X. G. Ren, C. G. Ning, J. K. Deng, S. F. Zhang, G. L. Su, F. Huang and G. Q. Li, Phys. Rev. Lett., 2005, 94, 163201.

[99] (a) J. Delhalle, J. P. Denis, M. S. Deleuze, J. Riga and M. Dosiere, Chem. Phys. Lett., 1993, 210, 21; (b) M. S. Deleuze, J. Delhalle and B .T. Pickup, Chem. Phys., 1993, 175, 427; (c) J. Riga, J. Delhalle, M. S. Deleuze and J. J. Verbist, Surf. Int. Anal., 1994, 22, 507; (d) I. Flamant, D. H. Mosley, M. S. Deleuze, J. M. Andre and J. Delhalle, Int. J. Quantum Chem., 1994, 528, 469; (e) A. S. Duwez, S. Di Paolo, J. Ghijsens, J. Riga, M. S. Deleuze and J. Delhalle, J. Phys. Chem. B, 1997, 101, 884. 


\section{Figure captions}

\section{Figure 1}

Geometric structure of the puckered conformer $\left(\mathrm{C}_{\mathrm{s}}\right)$ of cyclopentene.

\section{Figure 2}

Molecular orbital contours of cyclopentene in its $\mathrm{C}_{\mathrm{s}}$ form (B3LYP/aug-cc-pVTZ results).

\section{Figure 3}

Comparison of the (a) He I and (b) He II photoelectron spectra of cyclopentene with (c) $\mathrm{ADC}(3) / \mathrm{cc}-\mathrm{pVDZ}$ and (d) ADC(3)/DZP+ simulations (Convolution performed using a FWHM parameter of $0.6 \mathrm{eV})$.

\section{Figure 4}

The valence shell electron binding energy spectra (BES) of cyclopentene in the range 2-34 eV at azimuthal angle $\phi=1$ and $\phi=8^{\circ}$ (EMS results [56]).

\section{Figure 5}

Comparison of experimental and theoretical orbital densities in momentum space for band $\mathbf{I}$, due to the $12 \mathrm{a}^{\prime}$ level of cyclopentene.

\section{Figure 6}

Comparison of experimental and theoretical orbital densities in momentum space for band II, due to the $7 a^{\prime \prime}, 11 a^{\prime}, 10 a^{\prime}, 6 a^{\prime \prime}$ and $9 a^{\prime}$ set of orbitals of cyclopentene.

\section{Figure 7}

Comparison of experimental and theoretical orbital densities in momentum space for band III, due to the $5 \mathrm{a}^{\prime \prime}$ level of cyclopentene. 


\section{Figure 8}

Comparison of experimental and theoretical orbital densities in momentum space for band $\mathbf{I V}$, due to the $8 \mathrm{a}^{\prime}$ and $7 \mathrm{a}^{\prime}$ set of orbitals.

\section{Figure 9}

Comparison of experimental and theoretical orbital densities in momentum space for band $\mathbf{V}$, due to the $7 \mathrm{a}$ ' satellite line at $17.3 \mathrm{eV}(\Gamma=0.12)$, and to the $4 \mathrm{a}^{\prime \prime}$ and $6 \mathrm{a}^{\prime}$ set of orbitals of cyclopentene.

\section{Figure 10}

Detailed comparison of experimental and theoretical orbital densities in momentum space for band VI, due to $3 \mathrm{a}^{\prime \prime}$ and $5 \mathrm{a}^{\prime}$ set of orbitals of cyclopentene.

\section{Figure 11}

Detailed comparison of experimental and theoretical orbital densities in momentum space for band VII, due to $4 \mathrm{a}^{\prime}$ level of cyclopentene. 
Table 1 - Selected B3LYP/aug-cc-pVTZ optimized geometrical parameters for the $\mathrm{C}_{\mathrm{s}}$ conformers of cyclopentene. Bond lengths are in angstroms. Bond $(\theta)$ and puckered $(\varphi)$ angles are in degrees.

\begin{tabular}{|c|c|c|c|c|c|}
\hline parameter & MM4 $^{\mathrm{a}}$ & $\begin{array}{c}\text { MP2(full)/ } \\
6-31 \mathrm{G}^{* \mathrm{~b}}\end{array}$ & $\begin{array}{c}\text { B3LYP/ } \\
\text { aug-cc-pVT } \\
\mathrm{Z}^{\mathrm{c}}\end{array}$ & $\begin{array}{c}\text { Microwave } \\
\text { spectrum }^{\mathrm{d}}\end{array}$ & $\begin{array}{c}\text { gas-phase } \\
\text { electron } \\
\text { diffraction }^{\mathrm{e}}\end{array}$ \\
\hline $\mathrm{C}_{1}-\mathrm{C}_{2}$ & 1.344 & 1.340 & 1.329 & & $1.343 \pm 0.01$ \\
$\mathrm{C}_{2}-\mathrm{C}_{3}$ & 1.518 & 1.507 & 1.508 & 1.518 & 1.519 \\
$\mathrm{C}_{3}-\mathrm{C}_{4}$ & 1.552 & 1.540 & 1.549 & & 1.546 \\
$\theta\left(\mathrm{C}_{1}, \mathrm{C}_{2}, \mathrm{C}_{3}\right)$ & 111.3 & 111.5 & 112.2 & & $111.0 \pm 2$ \\
$\theta\left(\mathrm{C}_{2}, \mathrm{C}_{3}, \mathrm{C}_{4}\right)$ & 103.6 & 102.5 & 103.1 & & 103.0 \\
$\theta\left(\mathrm{C}_{3}, \mathrm{C}_{4}, \mathrm{C}_{5}\right)$ & 104.0 & 105.1 & 105.6 & 106.3 & 104.0 \\
Puckering & 24.6 & 26.3 & 19.4 & $22.3 \pm 2$ & $29.0 \pm 2.5$ \\
angle $(\varphi)^{\mathrm{f}}$ & & & & & \\
\hline
\end{tabular}

a. See ref. 79

b. See ref. 80

c. present work

d. See ref. 25

e. See ref. 29

f. Puckering angle is angle between the $\mathrm{C}_{3} \mathrm{C}_{4} \mathrm{C}_{5}$ plane and the $\mathrm{C}_{5} \mathrm{C}_{1}=\mathrm{C}_{2} \mathrm{C}_{3}$ plane 
Table 2 -Assignment of the experimentally available ionization spectra of cyclopentene, using HF, DFT/B3LYP, OVGF and ADC(3) ionization energies. Binding energies are given in eV. Spectroscopic strengths (or pole strengths $\Gamma_{n}$ ) are given in bracket.

\begin{tabular}{|c|c|c|c|c|c|c|c|c|c|c|}
\hline Level & orbital & $\begin{array}{c}\mathrm{HF} / \\
\text { aug-cc-pVTZ }\end{array}$ & $\begin{array}{l}\mathrm{ADC}(3) / \\
\text { cc-pVDZ }^{\mathrm{a}}\end{array}$ & $\begin{array}{c}\mathrm{ADC}(3) / \\
\mathrm{DZP}+{ }^{\mathrm{a}}\end{array}$ & $\begin{array}{c}\text { OVGF/ } \\
\text { Aug-cc-pVTZ }\end{array}$ & $\begin{array}{c}\text { B3LYP/ } \\
\text { aug-cc-pVTZ }\end{array}$ & $\mathrm{PES}^{\mathrm{b}}$ & $\mathrm{PES}^{\mathrm{c}}$ & PES $^{\mathrm{d}}$ & $\mathrm{EMS}^{\mathrm{e}}$ \\
\hline 1 & $12 \mathrm{a}^{\prime}$ & 9.267 & $9.135(0.907)$ & $9.128 \quad(0.906)$ & $9.267(0.910)$ & 6.649 & 9.18 & 9.20 & & 9.15 \\
\hline 2 & $7 a^{\prime \prime}$ & 12.848 & $11.955(0.904)$ & $11.971(0.904)$ & $11.933(0.904)$ & 9.308 & 11.6 & 11.6 & & \\
\hline 3 & $11 \mathrm{a}^{\prime}$ & 13.153 & $12.353(0.906)$ & $12.374(0.906)$ & $12.374(0.910)$ & 9.392 & 12.0 & 12.0 & & \\
\hline 4 & $10 \mathrm{a}^{\prime}$ & 13.250 & $12.207(0.901)$ & $12.232(0.900)$ & $12.260(0.901)$ & 9.553 & 12.2 & 12.2 & & 11.95 \\
\hline 5 & 6a" & 13.392 & $12.219(0.902)$ & $12.259(0.901)$ & $12.192(0.902)$ & 9.501 & 12.6 & 12.6 & & \\
\hline 6 & $9 a^{\prime}$ & 14.483 & $13.230(0.901)$ & $13.268(0.901)$ & $13.236(0.902)$ & 10.384 & 13.1 & 13.08 & & \\
\hline 7 & $5 \mathrm{a}^{\prime \prime}$ & 14.943 & $13.882(0.895)$ & $13.906(0.895)$ & $14.009(0.904)$ & 10.885 & 14.0 & 14.0 & & 14.19 \\
\hline 8 & $8 a^{\prime}$ & 17.225 & $15.964(0.877)$ & $15.980(0.878)$ & $15.945(0.899)$ & 12.752 & 15.8 & 15.8 & & \\
\hline 9 & $7 a^{f^{f}}$ & 18.277 & $\begin{array}{l}16.503(0.374) \\
16.689(0.362) \\
17.262(0.124)\end{array}$ & $\begin{array}{l}16.538(0.374) \\
16.718(0.361) \\
17.283(0.123)\end{array}$ & $16.659(0.874)$ & 13.573 & $16.5^{\mathrm{g}}$ & $16.5^{\mathrm{g}}$ & & 16.05 \\
\hline 10 & $4 \mathrm{a}^{\prime \mathrm{f}}$ & 19.642 & $17.614(0.678)$ & $\begin{array}{l}17.658(0.607) \\
17.747(0.128)\end{array}$ & $17.757(0.866)$ & 14.397 & & $17.3^{\mathrm{g}}$ & & 18.30 \\
\hline 11 & $6 \mathrm{a}^{\mathrm{a}^{\mathrm{f}}}$ & 21.377 & $\begin{array}{l}18.949(0.103) \\
19.145(0.637)\end{array}$ & $19.193(0.680)$ & & 15.651 & & $19.0^{\mathrm{g}}$ & 18.75 & \\
\hline 12 & $3 a^{\prime \prime f}$ & 25.970 & $\begin{array}{l}22.709(0.120) \\
22.984(0.346)\end{array}$ & $\begin{array}{l}22.737(0.149) \\
23.004(0.254)\end{array}$ & & 19.202 & & $22.0^{\mathrm{g}}$ & 22.04 & 22.30 \\
\hline 13 & $5 \mathrm{a}^{\mathrm{ff}}$ & 26.182 & $\begin{array}{l}22.586(0.222) \\
22.621(0.185)\end{array}$ & $\begin{array}{l}22.410(0.131) \\
22.682(0.204)\end{array}$ & & 19.284 & & & & \\
\hline 14 & $4 a^{f^{f}}$ & 30.667 & $\begin{array}{l}26.144(0.044) \\
26.338(0.040)\end{array}$ & $\begin{array}{l}27.059(0.030) \\
27.191(0.030)\end{array}$ & & 22.887 & & & 26.0 & 25.85 \\
\hline
\end{tabular}




\begin{abstract}
a. This work
b. See ref. 62(a)

c. See ref. $62(\mathrm{~b})$

d. See ref. 62(c)

e. See ref. 47
\end{abstract}

f. Breakdown of the orbital picture of ionization. Additional ADC(3)/cc-pVDZ shake-up lines: 7a' $21.015(0.019)$, 4a" 17.088 (0.044), 4a" 17.754 (0.057), 4a" 18.568 (0.059), 4a" 21.415 (0.013), 4a" 21.486 (0.008); 6a' 19.495 (0.038), 6a' 19.925 (0.017), 6a' 20.601 (0.039), 6a' 20.697 (0.040), 6a' 20.879 (0.008); 3a" 20.461 (0.007), 3a" 20.974 (0.007), 3a" 21.054 (0.018), 3a" 21.956 (0.009), 3a" 22.018 (0.028), 3a" 22.171 (0.019), 3a" 22.223 (0.010), 3a" 22.413 (0.023), 3a" 22.614 (0.019), 3a" 22.693 (0.037), 3a" 22.771 (0.050), 3a" 23.385 (0.014), 3a" 23.437 (0.013), 3a" 23.680 (0.010), 3a" 23.796 (0.025), 3a" 23.889 (0.022); 5a' 21.402 (0.006), 5a' 21.984 (0.007), 5a' 22.316 (0.023), 5a' 22.383 (0.060), 5a' 22.407 (0.024), 5a' 22.696 (0.022), 5a' 22.717 (0.013), 5a' 22.972 (0.019), 5a' 23.374 (0.005), 5a' 23.729 (0.017), 5a' 23.850 (0.010), 5a' 24.059 (0.063), 5a' 24.307 (0.006), 5a' 24.443 (0.053), 5a' 24.514 (0.019), 5a' 24.550 (0.033), 5a' 24.786 (0.005), 5a' 25.092 (0.005), 5a' 25.192 (0.005); 4a' 24.379 (0.007), 4a' 25.524 (0.005), 4a' 25.544 (0.006), 4a' 25.817 (0.020), 4a' 25.997 (0.007), 4a' 26.004 (0.013), 4a' 26.089 (0.012), 4a' 26.114 (0.021), 4a' 26.279 (0.025), 4a' 26.343 (0.006), 4a' 26.412 (0.017), 4a' 26.451 (0.008), 4a' 26.546 (0.007), 4a' 26.669 (0.007), 4a' 26.691 (0.037), 4a' 26.804 (0.006), 4a' 26.818 (0.010), 4a' 26.848 (0.032), 4a' 26.914 (0.008), 4a' 26.928 (0.010), 4a' 26.941 (0.007), 4a' 26.976 (0.008), 4a' 26.991 (0.014), 4a' 27.039 (0.025), 4a' 27.086 (0.009), 4a' 27.145 (0.017), 4a' 27.206 (0.010), 4a' 27.227 (0.025), 4a' 27.260 (0.007), 4a' 27.310 (0.011), 4a' 27.315 (0.014), 4a' 27.440 (0.012), 4a' 27.520 (0.022), 4a' 27.621 (0.009), 4a' 27.677 (0.007), 4a' 27.771 (0.005), 4a' 27.780 (0.012), 4a' 27.943 (0.007), 4a' 27.974 (0.007), 4a' 28.084 (0.006). Additional ADC(3)/DZP+ shake-up lines: 7a' 21.020 (0.025), 4a" 17.109 (0.040), 4a" 18.586 (0.061, 4a" 21.514 (0.016); 6a' 18.728 (0.009), 6a' 18.981 (0.076), 6a' 19.547 (0.023), 6a' 20.454 (0.007), 6a' 20.640 (0.005), 6a' 20.687 (0.029), 6a' 20.759 (0.043). 3a" 21.048 (0.022), 3a" 21.714 (0.007), 3a" 21.891 (0.010), 3a" 22.519 (0.006), 3a" 22.664 (0.073), 3a" 22.712 (0.021), 3a" 22.785 (0.059), 3a" 22.858 (0.014), 3a" 23.045 (0.011), 3a" 23.403 (0.006), 3a" 23.448 (0.010), 3a" 23.462 (0.017), 3a" 23.540 (0.014), 3a" 23.837 (0.016), 3a" 23.850 (0.014), 3a" 23.952 (0.006), 3a" 
24.089 (0.007); 5a' 22.129 (0.016), 5a' 22.312 (0.012), 5a' 22.526 (0.006), 5a' 22.583 (0.075), 5a' 22.646 (0.007), 5a' 22.823 (0.064), 5a' 22.934 (0.018), 5a' 23.004 (0.019), 5a' 23.133 (0.016), 5a' 23.874 (0.013), 5a' 24.040 (0.064), 5a' 24.062 (0.008), 5a' 24.154 (0.006), 5a' 24.434 (0.066), 5a' 24.662 (0.007), 5a' 24.784 (0.006), 5a' 24.816 (0.005), 5a' 24.837 (0.006); 4a' 25.580 (0.010); 4a' 25.791 (0.006), 4a' 25.826 (0.016), 4a' 25.827 (0.006), 4a' 25.846 (0.005), 4a' 25.882 (0.009), 4a' 25.914 (0.011), 4a' 25.929 (0.005), 4a' 25.981 (0.007), 4a' 25.994 (0.016), 4a' 26.073 (0.009), 4a' 26.097 (0.011), 4a' 26.121 (0.007), 4a' 26.305 (0.008), 4a' 26.330 (0.008), 4a' 26.396 (0.014), 4a' 26.423 (0.011), 4a' 26.602 (0.007), 4a' 26.619 (0.006), 4a' 26.658 (0.016), 4a' 26.667 (0.014), 4a' 26.742 (0.007), 4a' 26.805 (0.007), 4a' 26.818 (0.019), 4a' 26.834 (0.020), 4a' 26.880 (0.006), 4a' 26.925 (0.008), 4a' 26.945 (0.008), 4a' 26.998 (0.012), 4a' 27.069 (0.008), 4a' 27.094 (0.013), 4a' 27.209 (0.009), 4a' 27.221 (0.007), 4a' 27.262 (0.014), 4a' 27.321 (0.005), 4a' 27.351 (0.007), 4a' 27.458 (0.010), 4a' 27.499 (0.005), 4a' 27.637 (0.006)

g. our assignment 


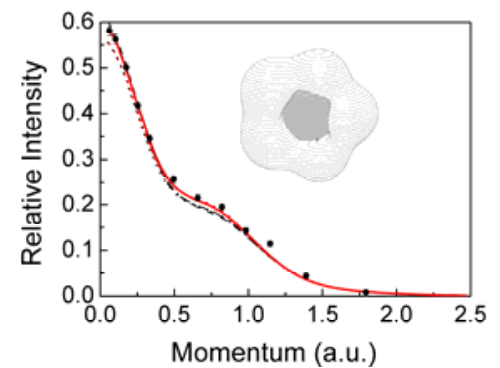

Dyson versus

Kohn-Sham orbital imaging in

momentum space

compared with

experiment 


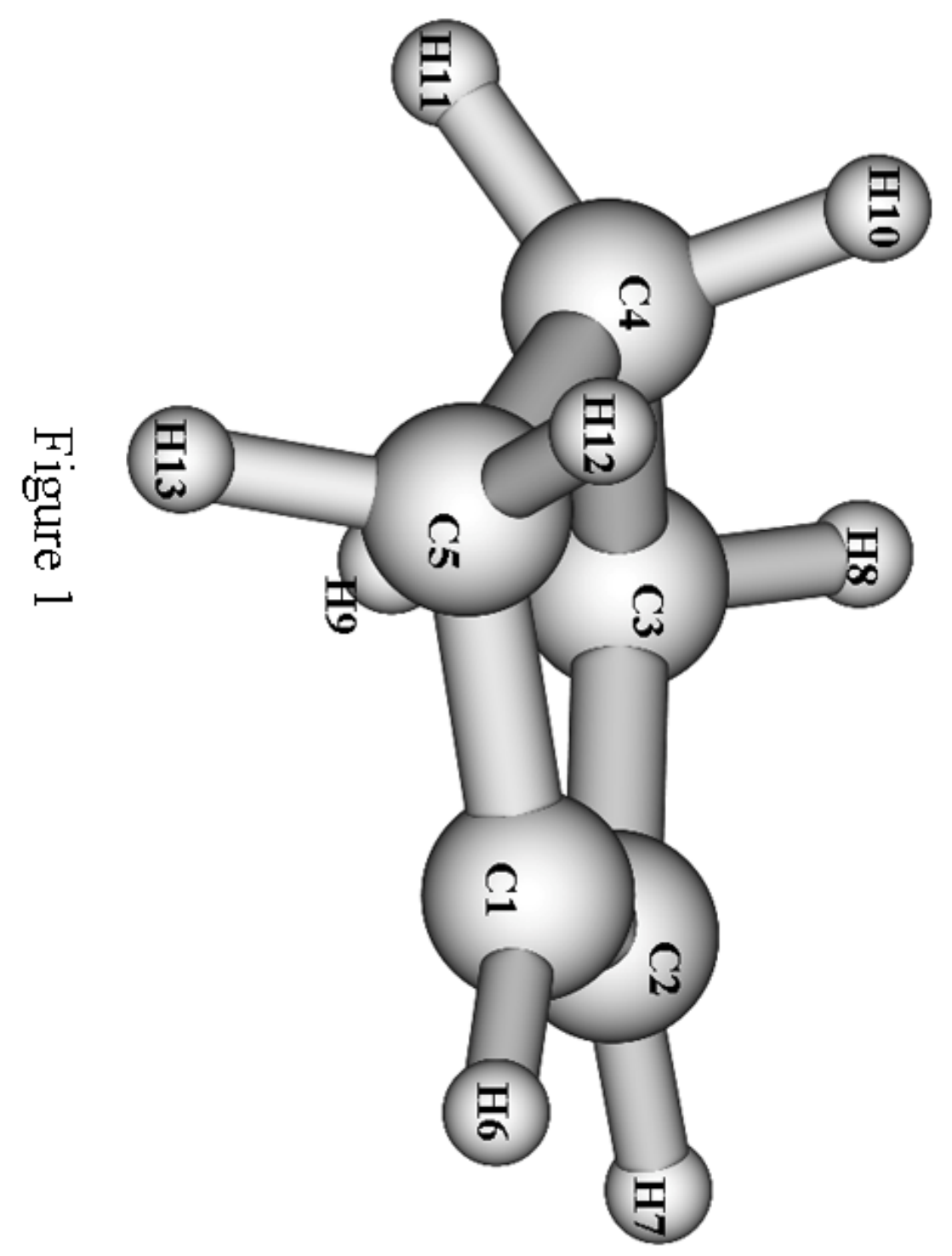


$12 \mathrm{a}^{\prime}(\mathbf{1})$

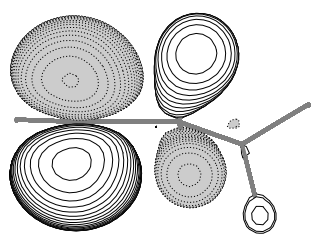

$9 \mathrm{a}^{\prime}(6)$

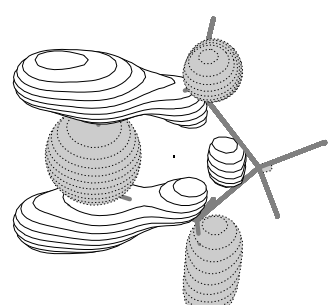

6a' (11)

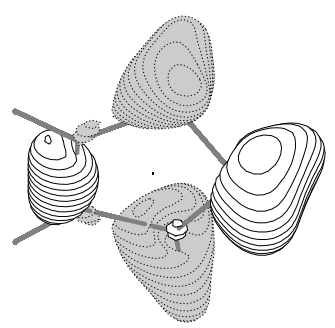

7a" (2)

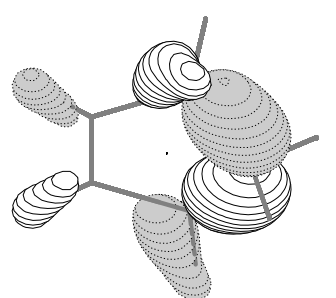

$5 a^{\prime \prime}(7)$

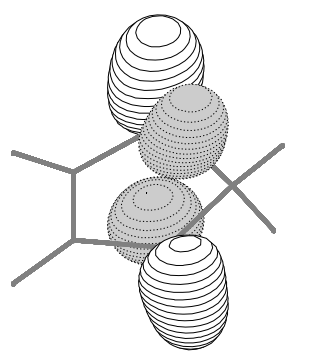

3a" (12)

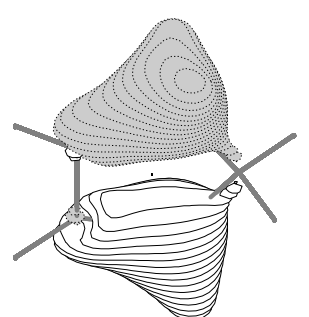

$11 a^{\prime}(3)$

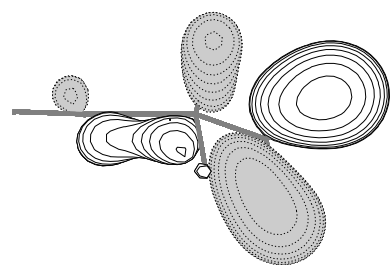

$8 a^{\prime}(8)$

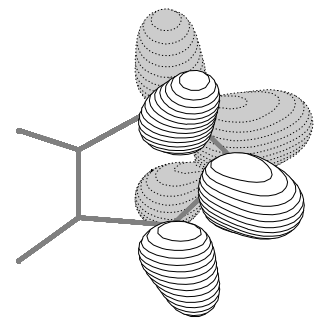

$5 \mathrm{a}^{\prime}(13)$

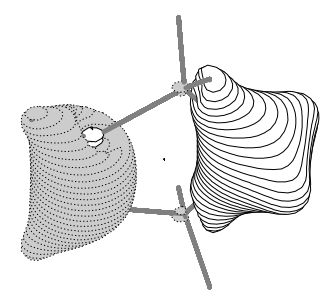

Figure 2 6a" (5)

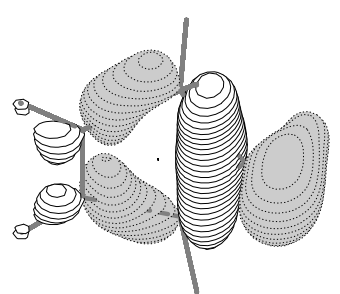

$7 a^{\prime}(9)$

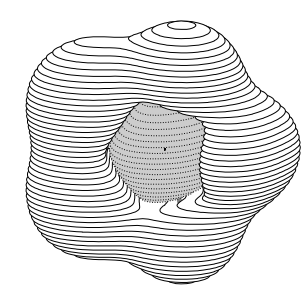

$4 a^{\prime}(14)$

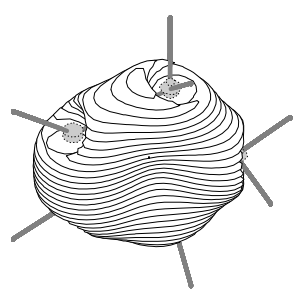

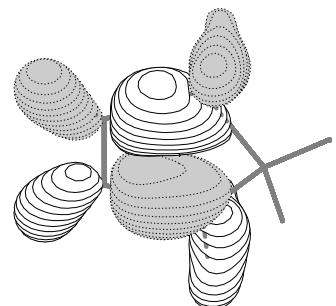

4a" (10)

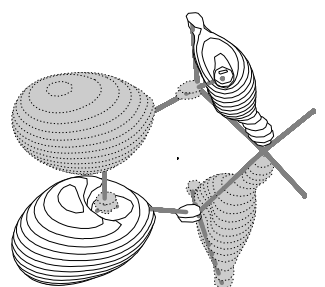




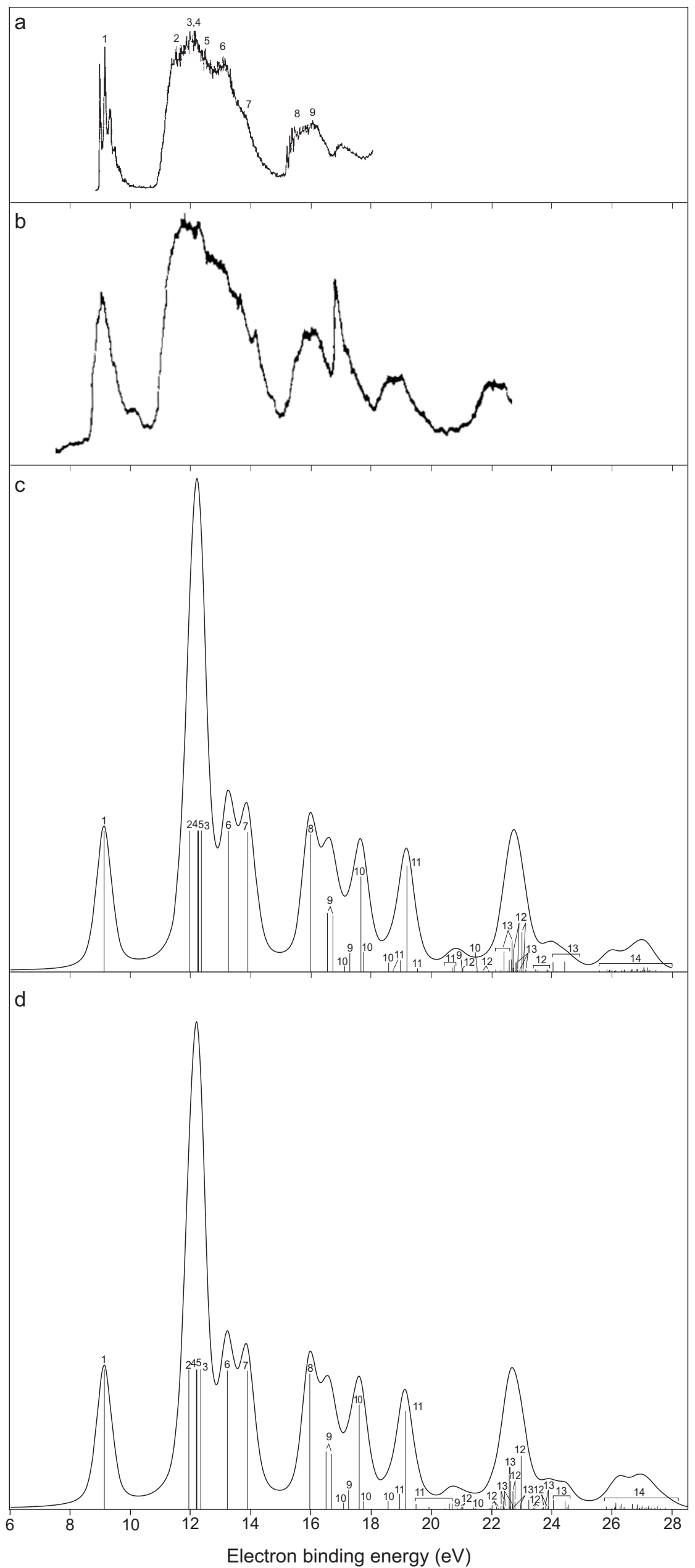

Figure 3 


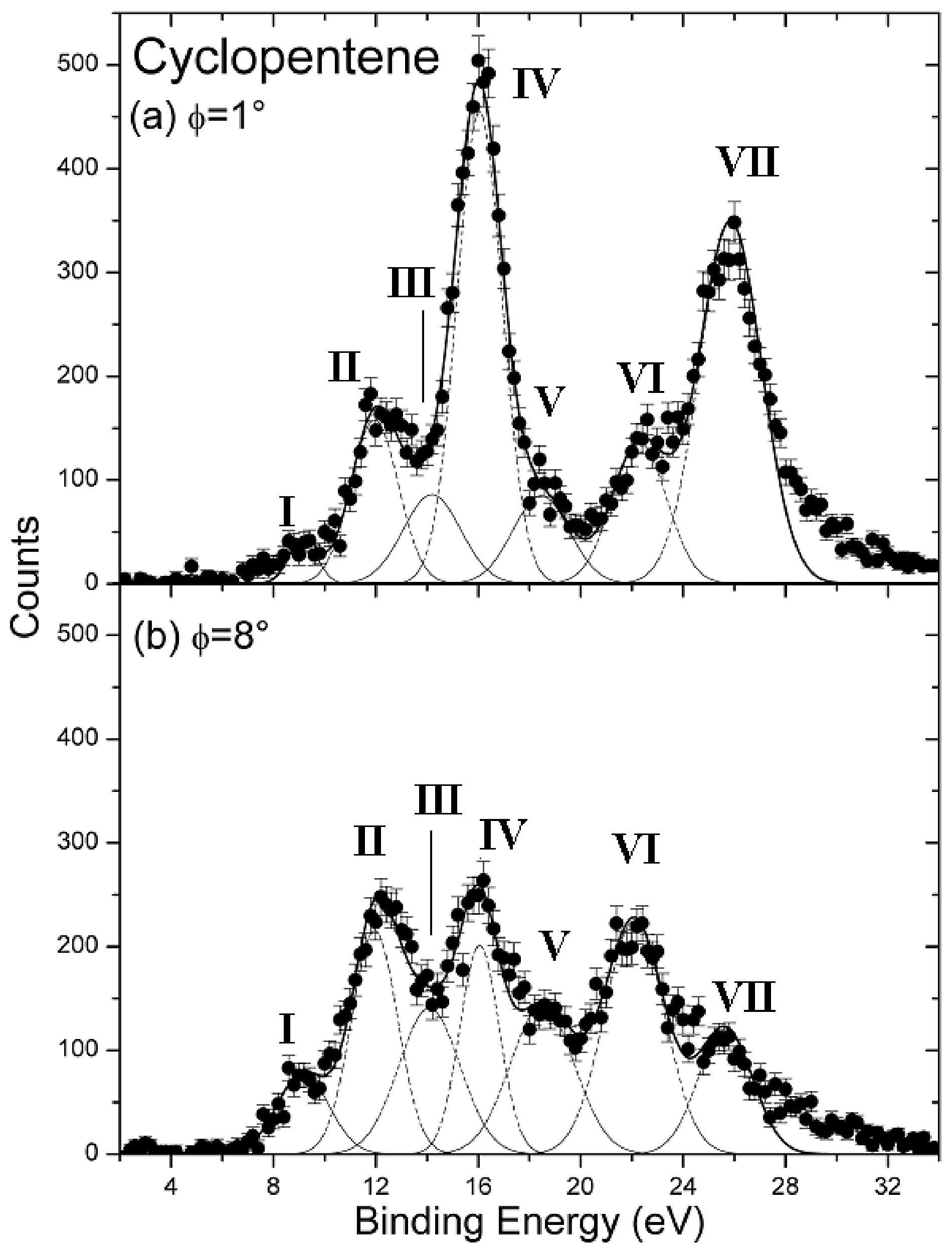

Figure 4 


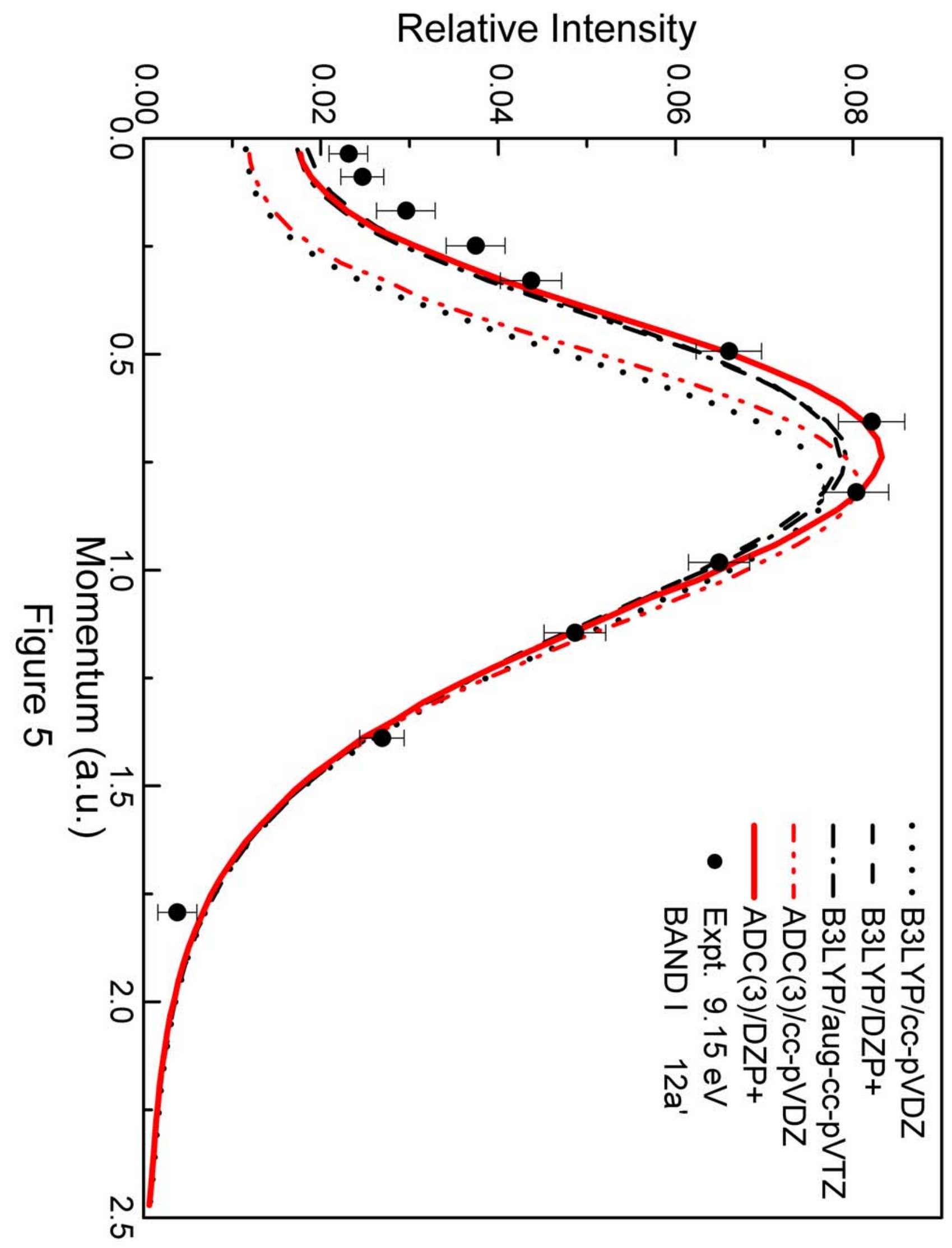




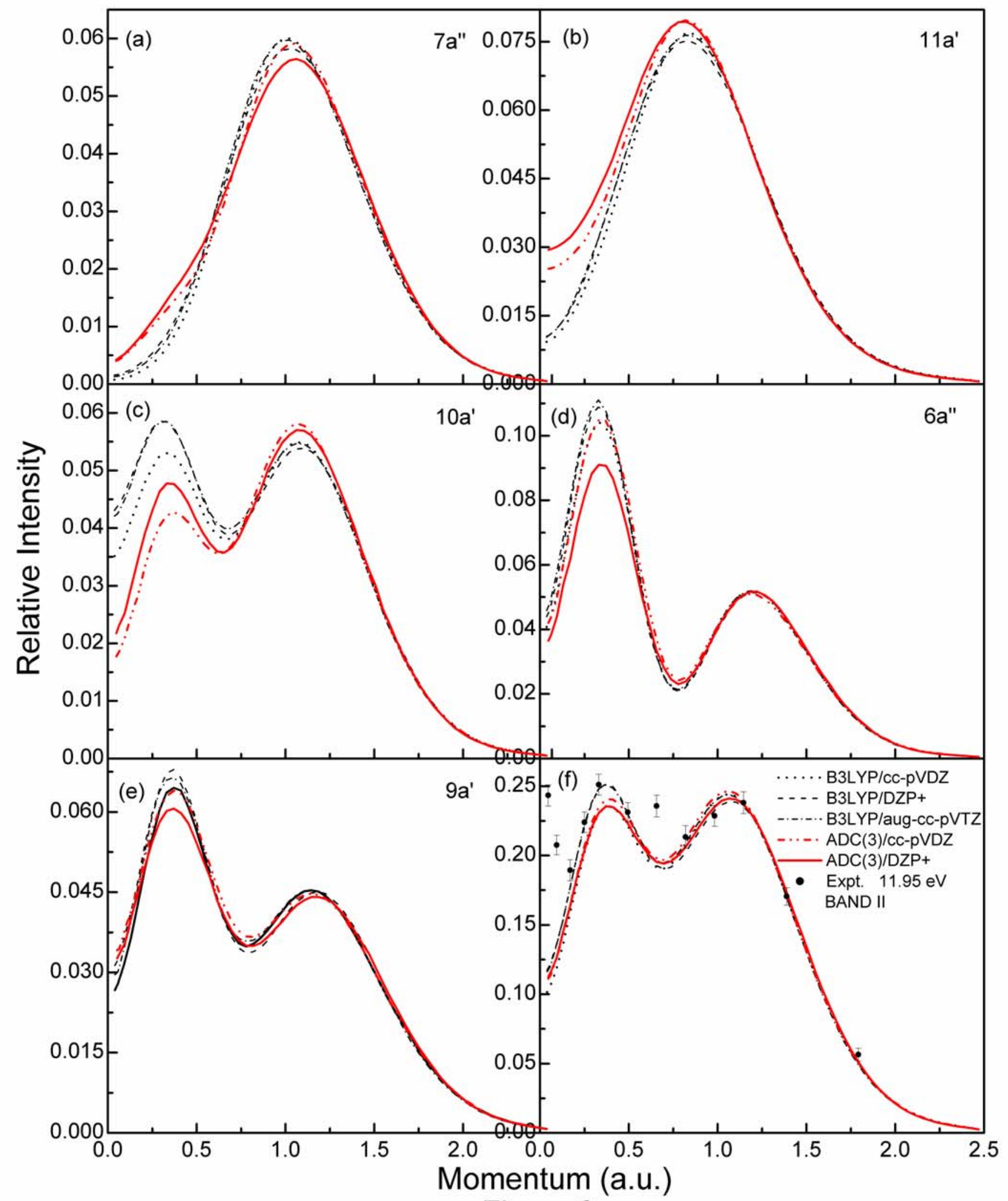

Figure 6 


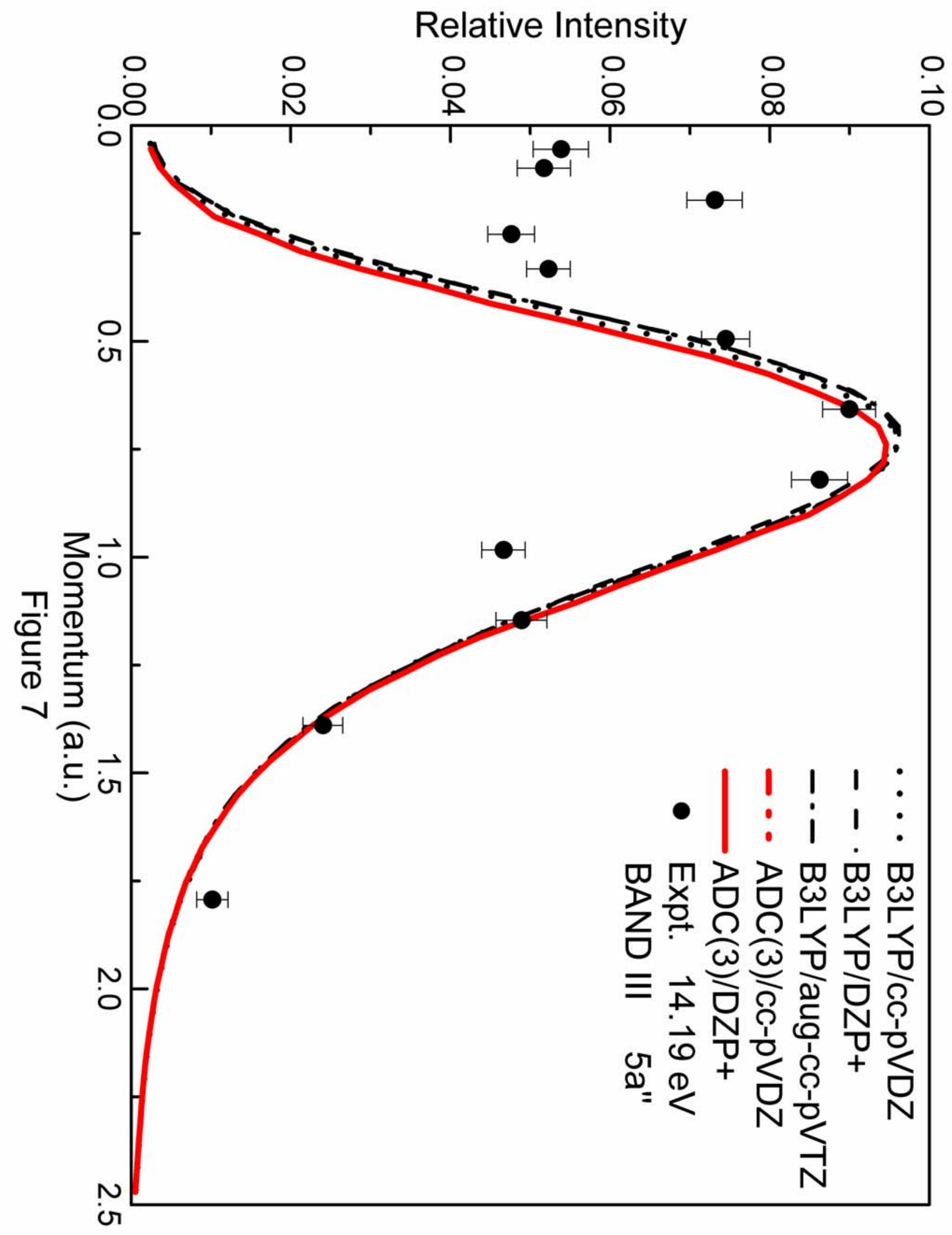




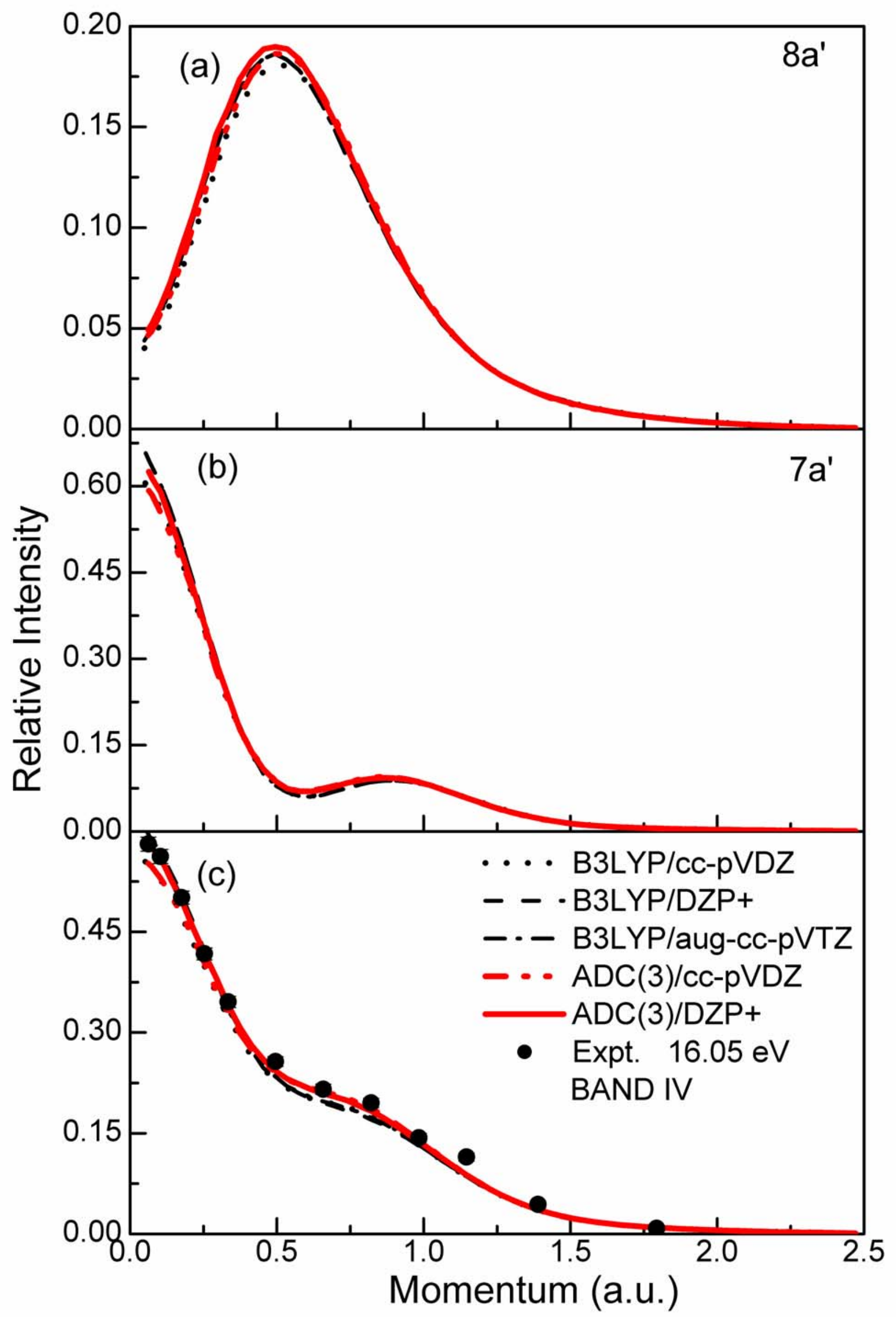

Figure 8 


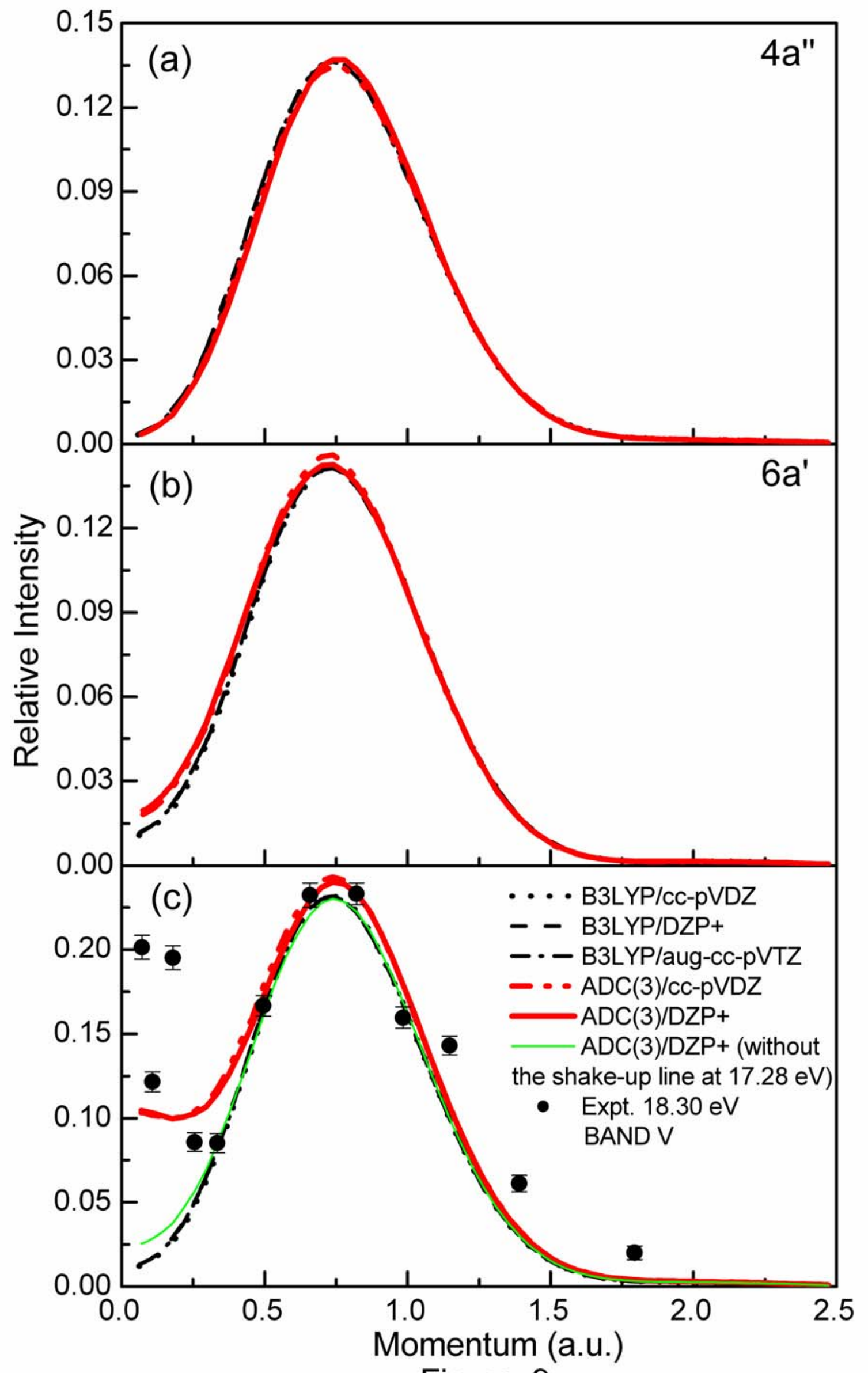

Figure 9 


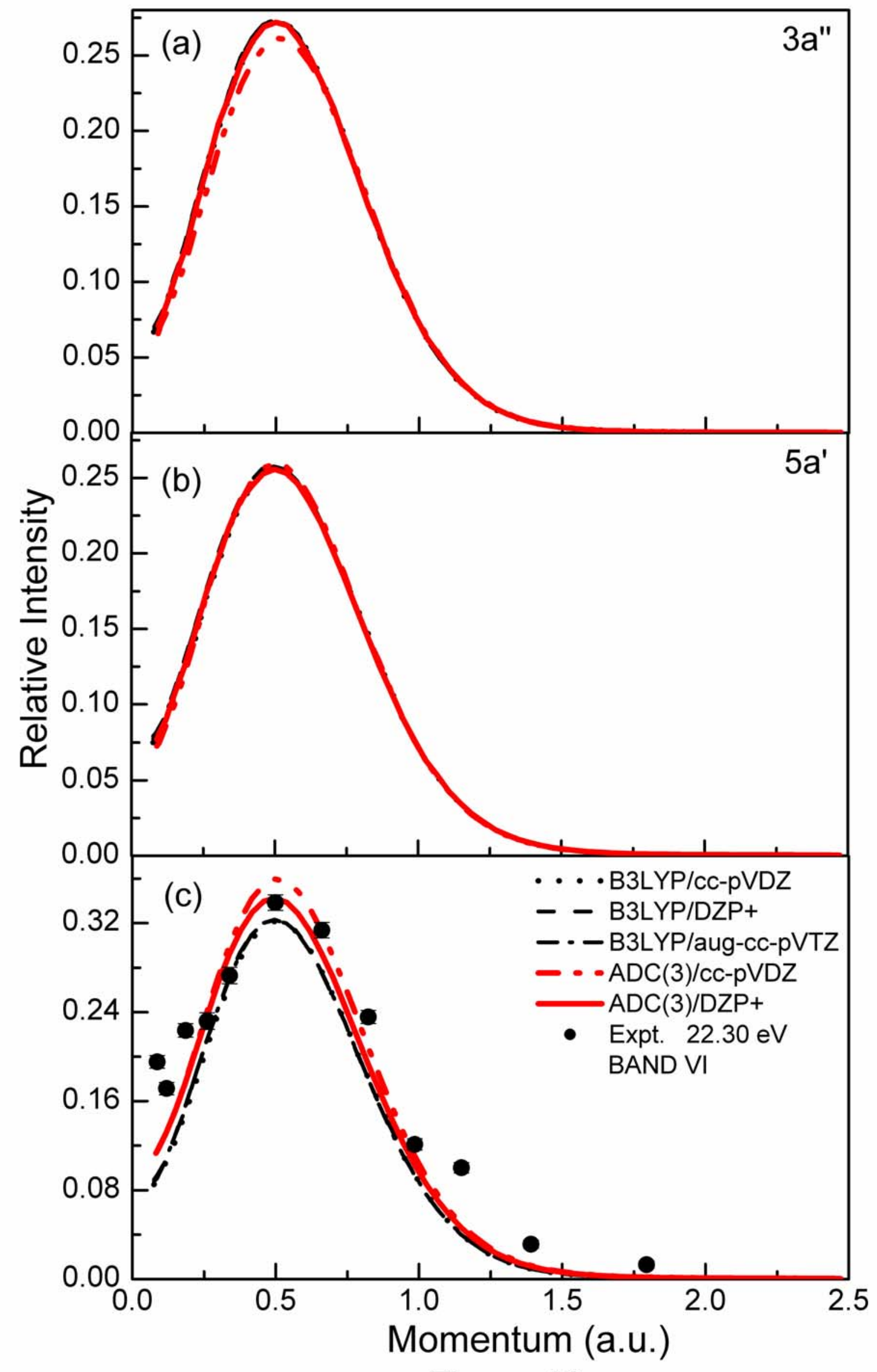

Figure 10 


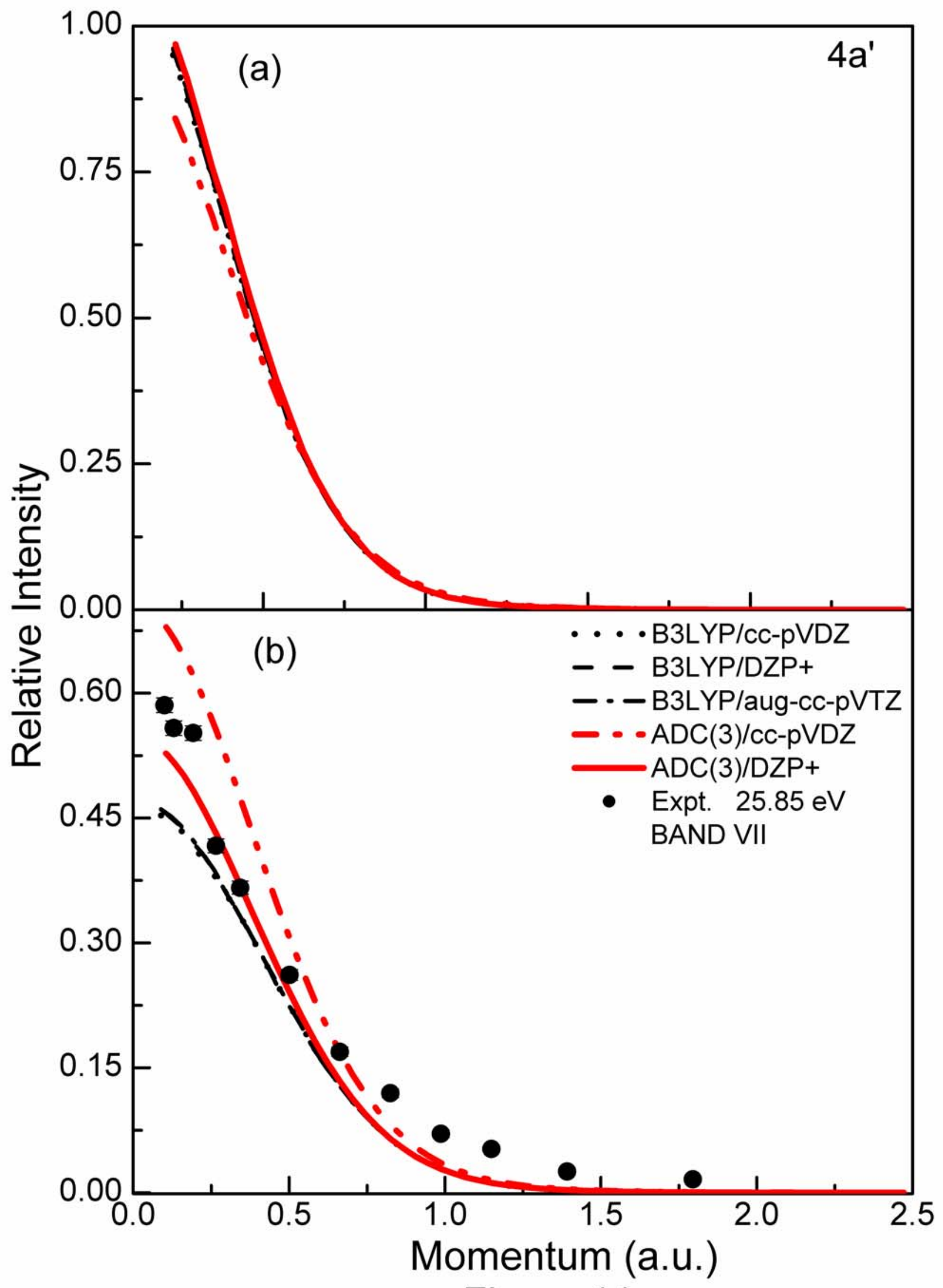

Figure 11 